ARTICLE

DOI: $10.1038 / s 41467-018-04987-y$

\title{
FABP4 as a key determinant of metastatic potential of ovarian cancer
}

Kshipra M. Gharpure ${ }^{1}$, Sunila Pradeep ${ }^{1,2}$, Marta Sans (10 ${ }^{3}$, Rajesha Rupaimoole ${ }^{4}$, Cristina Ivan (i) ${ }^{5,6}$, Sherry Y. Wu' ${ }^{1}$, Emine Bayraktar ${ }^{1}$, Archana S. Nagaraja ${ }^{1}$, Lingegowda S. Mangala ${ }^{1,5}$, Xinna Zhang ${ }^{5}$, Monika Haemmerle7, Wei Huㅜ, Cristian Rodriguez-Aguayo (10 5,6, Michael McGuire ${ }^{1}$, Celia Sze Ling Mak', Xiuhui Chen', Michelle A. Tran', Alejandro Villar-Prados' ${ }^{1}$, Guillermo Armaiz Pena ${ }^{8}$, Ragini Kondetimmanahalli ${ }^{9}$, Ryan Nini (10 ${ }^{10}$, Pranavi Koppula ${ }^{11}$, Prahlad Ram ${ }^{12}$, Jinsong Liu ${ }^{13}$, Gabriel Lopez-Berestein ${ }^{5,6}$, Keith Baggerly ${ }^{14}$, Livia S. Eberlin ${ }^{3} \&$ Anil K. Sood $1,5,15$

The standard treatment for high-grade serous ovarian cancer is primary debulking surgery followed by chemotherapy. The extent of metastasis and invasive potential of lesions can influence the outcome of these primary surgeries. Here, we explored the underlying mechanisms that could increase metastatic potential in ovarian cancer. We discovered that FABP4 (fatty acid binding protein) can substantially increase the metastatic potential of cancer cells. We also found that miR-409-3p regulates FABP4 in ovarian cancer cells and that hypoxia decreases miR-409-3p levels. Treatment with DOPC nanoliposomes containing either miR-409-3p mimic or FABP4 siRNA inhibited tumor progression in mouse models. With RPPA and metabolite arrays, we found that FABP4 regulates pathways associated with metastasis and affects metabolic pathways in ovarian cancer cells. Collectively, these findings demonstrate that FABP4 is functionally responsible for aggressive patterns of disease that likely contribute to poor prognosis in ovarian cancer.

\footnotetext{
${ }^{1}$ Department of Gynecologic Oncology and Reproductive Medicine, The University of Texas MD Anderson Cancer Center, Houston, Texas 77030, USA. ${ }^{2}$ Department of Obstetrics and Gynecology, Medical College of Wisconsin, Milwaukee, WI 53226, USA. ${ }^{3}$ Department of Chemistry, The University of Texas at Austin, Austin, TX 78712, USA. ${ }^{4}$ Department of Pathology and Institute of RNA Medicine, Beth Israel Deaconess Medical Center Cancer Center, Harvard Medical School, Boston, MA 02215, USA. ${ }^{5}$ Center for RNA Interference and Non-Coding RNA, The University of Texas MD Anderson Cancer Center, Houston, 77030, Texas, USA. ${ }^{6}$ Department of Experimental Therapeutics, The University of Texas MD Anderson Cancer Center, Houston, TX 77030, USA. ${ }^{7}$ Martin-Luther-University Halle-Wittenberg, Institute of Pathology, 06112 Halle (Saale), Germany. ${ }^{8}$ Department of Pharmacology, Ponce Health Sciences University, Ponce 00716, Puerto Rico. ${ }^{9}$ The University of Texas at Austin, Austin, TX 78712, USA. ${ }^{10}$ Graduate School of Biomedical Sciences, The University of Texas MD Anderson Cancer Center, Houston, TX 77030, USA. ${ }^{11}$ Department of Experimental Radiation Oncology, The University of Texas MD Anderson Cancer Center, Houston, TX 77030, USA. ${ }^{12}$ Department of Systems Biology, The University of Texas MD Anderson Cancer Center, Houston, TX 77030, USA. ${ }^{13}$ Department of Pathology, Division of Pathology and Laboratory Medicine, The University of Texas MD Anderson Cancer Center, Houston, TX 77030 , USA. ${ }^{14}$ Department of Bioinformatics and Computational Biology, The University of Texas MD Anderson Cancer Center, Houston, TX 77030, USA.

15 Department of Cancer Biology, The University of Texas MD Anderson Cancer Center, Houston, TX 77030, USA. Correspondence and requests for materials should be addressed to A.K.S. (email: asood@mdanderson.org)
} 
P rimary cytoreductive surgery followed by adjuvant chemotherapy is the standard treatment for ovarian cancer. Several reports have established a link between residual disease after surgery and shorter overall and progression-free survival, as well as poor response to adjuvant chemotherapy ${ }^{1-3}$. Incomplete resection can result from the presence of numerous, dense nodules that simply cannot be removed, distant tumor metastasis, location of tumor near critical organs (e.g., porta hepatis), and extensive mesenteric involvement. Thus, while a surgeon's skill is important, residual disease could occur because the intrinsic biology of the tumor creates a highly metastatic and infiltrative disease pattern, making complete resection infeasible $e^{3,4}$. A large retrospective study showed that initial disease distribution could also affect patient survival despite aggressive cytoreductive efforts ${ }^{5}$. Considering the impact of residual disease on patient survival, many studies are now focusing on the development of predictive models for residual disease. However, currently there is little understanding of the biological mechanisms that could lead to residual disease in ovarian cancer. To address this knowledge gap, in the current study, we explored the underlying tumor biology responsible for the aggressive pattern of tumor metastasis.

We have previously shown that high expression of fatty acid binding protein 4 (FABP4) can be a reliable molecular predictor of residual disease in high-grade serous ovarian cancer ${ }^{4}$. Hence, we decided to investigate the mechanisms that lead to upregulation of FABP4 in ovarian cancer, as well as its downstream effects that lead to metastasis. FABP4 is implicated in atherosclerosis, diabetes, inflammatory response, and angiogenesis $^{6-12}$. In prostate and ovarian cancers, FABP4 acts as a key mediator between adipocytes and cancer progression ${ }^{13,14}$. However, FABP4 regulation and the functional implications of FABP4 overexpression in increasing the metastatic potential of ovarian cancer cells remain to be investigated.

Here, we examined the biological effects of increased FABP4 expression in ovarian cancer. Through extensive bioinformatics analyses, we identified hypoxia mediated downregulation of miR409-3p as a key regulator of FABP4. FABP4 siRNA or a miR-409$3 \mathrm{p}$ mimic led to significant inhibition of tumor metastasis. Downstream of FABP4, we observed that FABP4 can affect several metabolites as well as metastasis-related pathways in ovarian cancer. Collectively, this study provides an understanding of the causal biology of metastatic potential in ovarian cancer.

\section{Results}

Effects of FABP4 on tumor progression. To investigate the functional role of FABP4 in ovarian cancer progression, we first knocked down FABP4 in HeyA8 MDR ovarian cancer cells (which have high FABP4 expression) using small interfering RNA (siRNA). FABP4 silencing led to a significant reduction in both migration and invasion of HeyA8 MDR cancer cells (Fig. 1a, b, Supplementary Fig. $1 \mathrm{a}$ and b) $(p<0.01)$. Conversely, ectopic expression of FABP4 in A2780-ip1 ovarian cancer cells led to increased migration $(p<0.05$; Fig. 1c) and invasion of cancer cells $(p<0.01$; Fig. 1d, Supplementary Fig. 1c and d). The effect was consistent when tested using a second siRNA sequence against FABP4 (Supplementary Fig. 1e and f), as well as another ovarian cancer cell line (Ovcar 5; Supplementary Fig. 1g and h).

Next, we examined the effect of FABP4 on tumor progression in orthotopic mouse models of ovarian cancer. We first transfected A2780-ip1 cells that have low endogenous FABP4 levels with a lentivirus expressing either a control vector or FABP4-expressing vector. We then injected the cells into the ovaries of athymic nude mice. This orthotopic model mimics the ovarian cancer metastasis pattern seen in patients ${ }^{15,16}$. Compared with the control mice, mice injected with cells ectopically expressing FABP4 had a significantly higher tumor burden $(\sim 3-$ fold, $p<0.01$; Fig. 1e), as well as an increased number of distant metastatic nodules ( 3 -fold, $p<0.01$; Fig. 1 f). We examined hematoxylin and eosin-stained sections of tumor nodules from both groups. In the control group, the cancer cells did not invade normal organs (muscle, pancreas), however, after ectopic expression of FABP4, the cancer cells were found to be deeply invading the normal tissues (Fig. $1 \mathrm{~g}$ and Supplementary Fig 1i). The tumor metastasis rate with FABP4 overexpression was significantly higher at sites such as the diaphragm, liver, and pelvis, whereas tumors in the control mice were mainly observed at the site of injection (ovary) and in the mesentery (Fig. 1h). Expression of FABP4 was examined in the tumor sections using immunohistochemistry, and expression of FABP4 was higher in sections from the ectopic FABP4 group than in those from the control group (Supplementary Fig. 1j). We also noted a positive correlation between the expression of FABP4 and the invasive capacity of ovarian cancer cell lines in vitro (Supplementary Fig 1k). Cell lines with higher FABP4 expression (HeyA8 MDR and Ovcar 5) had higher invasive potential compared to A2780 and SKOV3 cells.

We next investigated the effects of FABP4 knockdown in orthotopic mouse models of ovarian cancer. We first injected HeyA8 MDR-Luc cells into the ovaries of nude mice. Seven days after cell injection, DOPC nanoliposomes containing control siRNA or FABP4 siRNA were administered intraperitoneally twice per week. The mice that received FABP4 siRNA had a significant decrease in aggregate tumor weight $(80 \%, p<0.01)$ as well as number of metastatic nodules $(75 \%, p<0.01)$ compared with control mice (Fig. 1i, j). The effect was also observed in the pattern of metastasis. The treatment group had a significantly lower rate of metastasis, and the control group had widespread metastases to the diaphragm, liver, spleen, lymph node, and pelvic areas (Fig. 1k). Bioluminescence images also supported the finding that FABP4 siRNA led to decreased metastasis in treated mice (Fig. 11). The treatment did not affect mouse body weight (Supplementary Fig. 2a). We then checked expression of FABP4 in the tumors collected from the control and treatment groups. FABP4 expression was significantly decreased after treatment with FABP4 siRNA, as observed in Western blot analysis (Supplementary Fig. 2b) and immunohistochemical staining (Supplementary Fig. 2c).

We also examined an additional orthotopic mouse model in which Ovcar 5 cells were injected directly into the ovary. Similar to the previous experiment, FABP4 siRNA treatment resulted in significant reduction in tumor weight $(p<0.05$; Supplementary Fig. 2d) and the number of nodules $(p<0.05$; Supplementary Fig. 2e). Metastasis was also reduced at various sites, including the peritoneum, mesentery, and diaphragm (Supplementary Fig. 2f). The treatment had no effect on the body weight of the mice (Supplementary Fig. 2g).

Upstream regulation of FABP4. Next, we investigated the potential mechanisms by which FABP4 is regulated in tumors. We first sought to determine whether there is any association between copy number and mRNA expression of FABP4. We examined The Cancer Genome Atlas (TCGA) datasets across platforms (Affymetrix, Agilent, RNASeqv2) and found no correlation between copy number and mRNA expression (Supplementary Fig. 3a). We also examined whether microRNAs (miRNAs) could regulate FABP4 levels. Our miRNA selection process is presented in Fig. 2a. We probed the TCGA dataset and selected samples from chemotherapy-naive cases of high-grade serous ovarian cancer. In these samples, we looked for miRNAs 
a

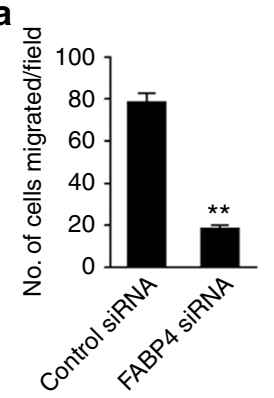

b

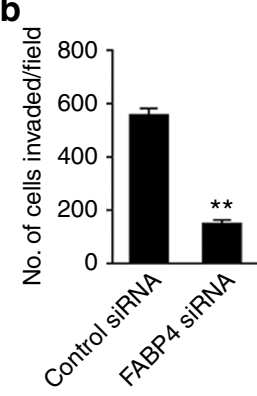

C

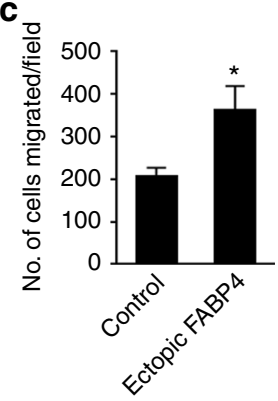

d

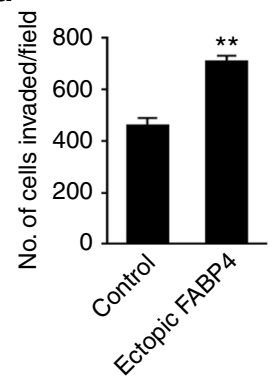

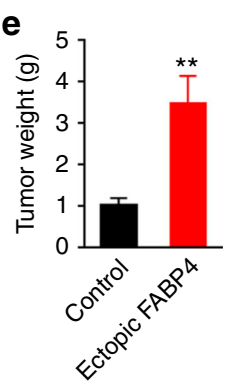

f
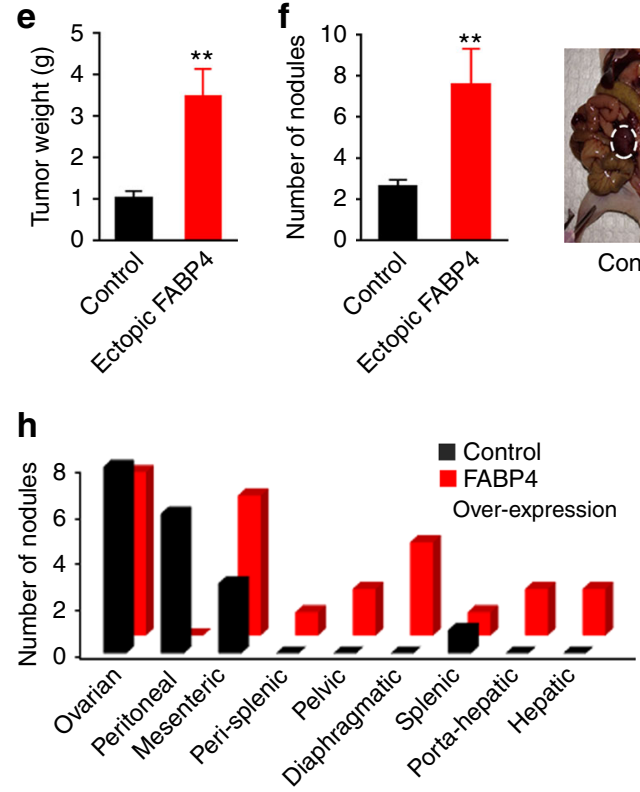

K

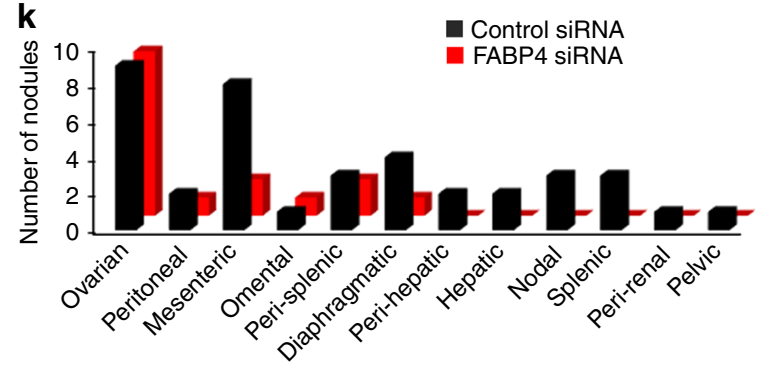

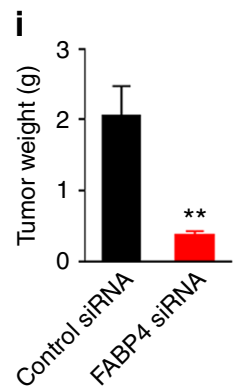

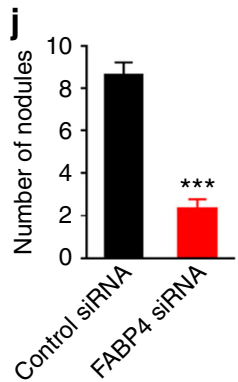

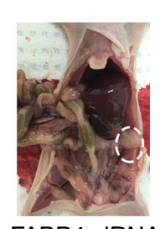

FABP4 SiRNA

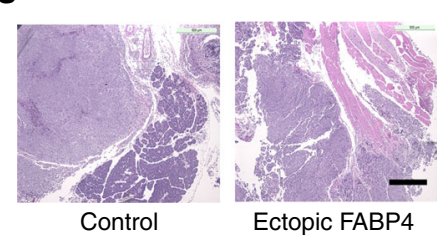

I
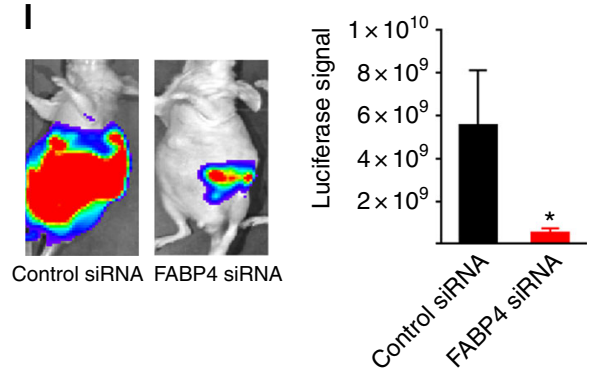

Fig. 1 Effects of FABP4 on ovarian tumor progression. $\mathbf{a}$, $\mathbf{b}$ Effect of knockdown of FABP4 on the $\mathbf{a}$ migration and $\mathbf{b}$ invasion of ovarian cancer cells (HeyA8 MDR). ${ }^{\star \star} p<0.01$. Effect of increased expression of FABP4 on the $\mathbf{c}$ migration and $\mathbf{d}$ invasion of cancer cells (A2780-ip1). ${ }^{\star} p<0.05 .{ }^{* \star} p<0.01$. e Aggregate mass of tumors in orthotopic mouse models involving A2780-ip1 cells transfected with control and FABP4 ectopic expression vectors ( $n=10$ mice per group, Student $t$ test). ${ }^{\star \star} p<0.01$. f Effect of ectopically expressed FABP4 on the number of nodules in orthotopic mouse models involving A2780-control and A2780-FABP4 ectopic expression ( $n=10$ mice per group, Student $t$ test). ${ }^{\star *} p<0.01$. Representative images of nodules are shown on the right. g Representative hematoxylin and eosin-stained sections of tumor tissues from mice injected with control A2780-ip1 cells or A2780-ip1 cells transfected with FABP4-expressing vector. $\mathbf{h}$ Distribution of metastatic nodules present in the groups. $\mathbf{i}$ Aggregate mass of tumors in orthotopic mouse models involving HeyA8 MDR cells. Mice were treated with control or FABP4 siRNA incorporated in DOPC liposomes $\left(n=10\right.$ mice per group). ${ }^{\star \star} p<0.01$. $\mathbf{j}$ Effect of knockdown of FABP4 on the number of metastatic nodules in HeyA8 MDR orthotopic mouse models. ${ }^{\star \star \star} p<0.001$. Representative images are shown on the right. $\mathbf{k}$ Distribution of metastatic nodules present in individual mice after treatment with control or FABP4 siRNA incorporated in DOPC liposomes. I Representative images of metastatic lesions revealed by luciferase imaging, and quantitative assessment of luciferase signal from mice treated with control or FABP4 siRNA encapsulated in DOPC liposomes. ${ }^{*} p<0.05$. Bars and errors bars represent the means and the corresponding SEMs for $n \geq 3$

that were associated with FABP4 levels. To narrow our search further, we used the maximum information coefficient score ${ }^{17}$, where a score of 0 indicates no association and a score of 1 indicates perfect association between a gene and a miRNA. Thirty-two miRNAs with a score $>0.2$ were selected (Supplementary Table 1). We then selected miRNAs from this group that were predicted to bind to the $3^{\prime}$ untranslated region (UTR) of
FABP4 by miRNA-gene interaction prediction software. Mir409-3p was predicted to target FABP4 by nine different prediction software programs.

Next, we transfected HeyA8 MDR cells with miR-409-3p mimic, which led to a significant decrease $(85 \%, p<0.01)$ in FABP4 expression (Fig. 2b). This effect was also observed at the protein level (Fig. 2c). Similar effect was observed in another cell 
a

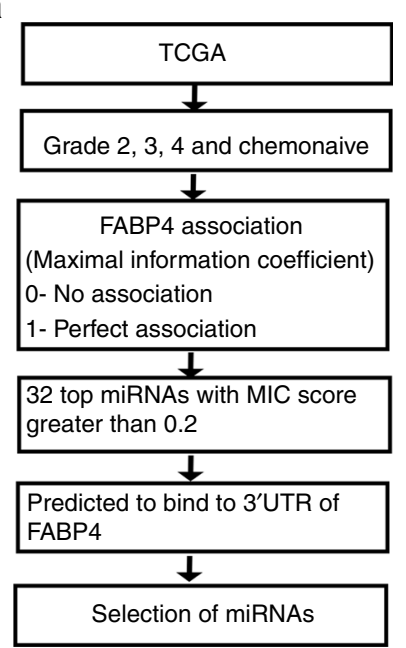

f

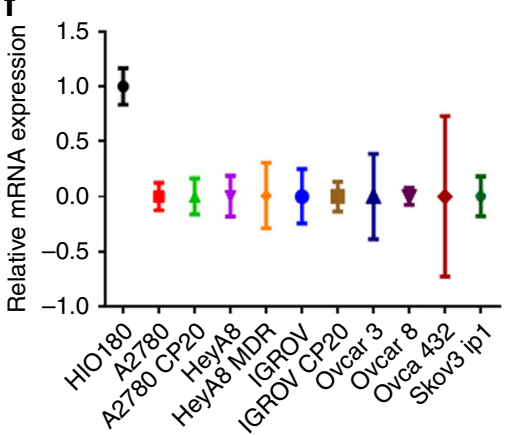

h

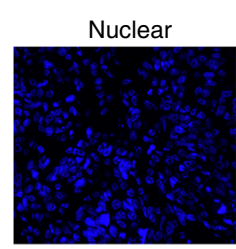

FABP4

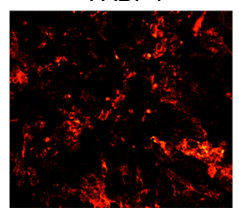

b

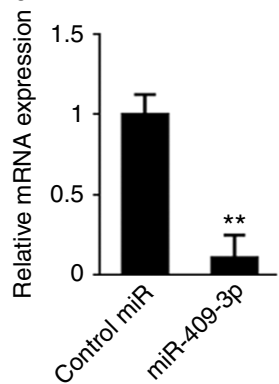

d

Mutated

Original

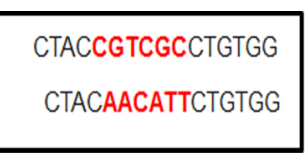

g

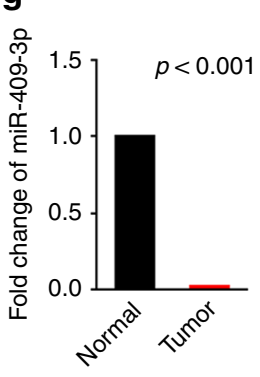

C

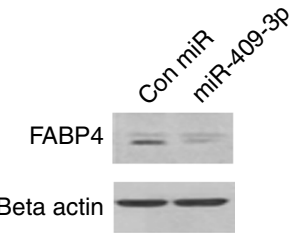

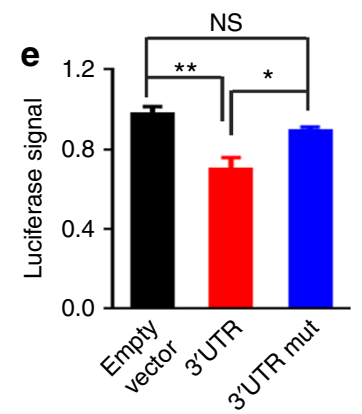

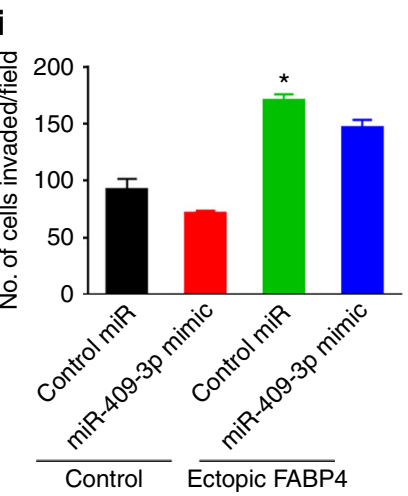

Fig. 2 Upstream regulation of FABP4. a Flowchart showing the methods used to identify microRNAs (miRNAs) that target FABP4 in ovarian cancer. $\mathbf{b}$ Effect of miR-409-3p mimic transfection specifically on FABP4 expression levels (RNA) in HeyA8 MDR cells. c Effect of miR-409-3p mimic transfection on FABP4 expression levels (protein) in HeyA8 MDR cells. d Original and mutated 3' untranslated region (UTR) sequence of FABP4, where miR-409-3p is predicted to bind. e Relative luciferase activity normalized to that of cells transfected with an empty control and cells transfected with control miRNA. HeyA8 MDR cells were transfected with empty control, wild-type FABP4 (red bar), or FABP4 with mutated 3'UTR (blue bar) and then transfected with a control miRNA mimic or a miR-409-3p mimic. Activity was measured $48 \mathrm{~h}$ after transfection. ${ }^{*} p<0.05$; ${ }^{\star *} p<0.01$; NS indicates not significant. f Expression of miR-409-3p in various ovarian cancer cell lines compared with normal ovarian cells (HIO180). $\mathbf{g}$ Expression of miR-409-3p in normal ovarian tissue and ovarian tumor samples in the GSE15190 dataset. $\mathbf{h}$ In situ hybridization for miR-409-3p (green) and FABP4 (red) in tumor tissues from patients with ovarian cancer $(n=8)$. Representative image is shown. $\mathbf{i}$ Invasion potential of SKOV3 ip1 cells was assessed in control cells or cells ectopically expressing FABP4 after transfection with control miRNA mimic or miR-409-3p mimic. Bars and errors bars represent the means and the corresponding SEMs for $n \geq 3$

line (Ovcar 5, $p<0.01$, Supplementary Fig. 3b; Ovca 432, $p<0.05$, Supplementary Fig. 3c). We also transfected ovarian cancer cells (Ovcar 3) with anti-miR-409-3p and then examined FABP4 expression. Inhibition of miR-409-3p led to a significant increase in FABP4 expression ( $p<0.05$; Supplementary Fig. $3 \mathrm{~d})$.

To determine whether miR-409-3p binds to the $3^{\prime}$ UTR of $F A B P 4$, we conducted a luciferase assay. The predicted binding site for miR-409-3p on FABP4 is presented in Supplementary Fig $3 \mathrm{e}$. We also mutated the binding site; the original sequence and mutated sequences are shown in Fig. 2d. In cells treated with a miR-409-3p mimic, the relative luciferase activity was significantly lower for cells transfected with FABP4 $3^{\prime}$ UTR than in cells transfected with an empty control. When FABP4's $3^{\prime}$ UTR binding site was mutated, abrogation of binding activity was 
observed (Fig. 2e), suggesting that miR-409-3p binds to the $3^{\prime}$ UTR of FABP4.

We next compared the expression of miR-409-3p in various ovarian cancer cell lines and non-transformed ovarian epithelial cells (HIO180). Compared with the control cells, all ovarian cancer cell lines showed low expression of miR-409-3p (Fig. 2f). We also analyzed the expression of FABP4 in these cell lines to determine the correlation between FABP4 and miR-409-3p at the basal level (Supplementary Fig 3f) and observed an inverse association between miR-409-3p and FABP4 in the cell lines. We then probed a Gene Expression Omnibus dataset $(\mathrm{GSE} 15190)^{18}$ and compared the expression of miR-409-3p in serous ovarian tumor tissues with that of normal ovarian tissue samples. The dataset revealed that miR-409-3p is expressed at significantly lower levels in ovarian tumors than in normal ovarian samples (Fig. 2g). We also examined 25 patient samples for correlations between miR-409-3p and FABP4 expression. In situ hybridization staining revealed a clear inverse association in the staining pattern of FABP4 and miR-409-3p (Fig. 2h).

To investigate whether the functions of miR-409-3p are mediated through FABP4, we performed a rescue experiment where we stably expressed a FABP4 construct lacking the $3^{\prime} \mathrm{UTR}$ in SKOV3ip1 cells so that it would not be sensitive to a miR-409$3 p$ mimic. Compared to controls, SKOV3ip1 cells with ectopically expressed FABP4 were significantly more invasive $(p<0.05)$. Treatment of the cell line ectopically expressing FABP4 with miR409-3p mimic did not result in a significant decrease in invasion (Fig. 2i), suggesting that FABP4 is an important target of miR-409-3p.

Effect of miR-409-3p on tumor progression and FABP4 expression. To determine whether miR-409-3p has tumorsuppressive properties, we transfected HeyA8 MDR cells with control miRNA or miR-409-3p mimic and conducted migration and invasion assays. Transfection of the miR-409-3p mimic led to a significant decrease in the migratory and invasive potential of cancer cells ( $p<0.01$; Fig. 3a, b). In contrast, reduction of miR409-3p with anti-miR-409-3p resulted in increased migration $(p<0.05)$ and invasion of cancer cells (Supplementary Fig. $4 \mathrm{a}$ and b). The effect of miR-409-3p mimic transfection was also observed with the Ovcar 5 cells. Migration (Supplementary Fig 4c) and invasion (Supplementary Fig 4d) potential of Ovcar 5 cells were significantly reduced after miR-409-3p transfection $\left({ }^{* *} p<0.01\right)$.

To examine the effects of miR-409-3p in vivo, we used the HeyA8 MDR orthotopic mouse model. Starting seven days after cell injection, DOPC nanoliposomes containing control miRNA or miR-409-3p were administered intraperitoneally twice weekly. Compared with controls, mice treated with miR-409-3p mimic showed significant reduction in tumor weight $(\sim 60 \%, p<0.01$; Fig. 3c) and number of tumor nodules ( $60 \%, p<0.01$; Fig. $3 d)$. The treated mice also showed a different pattern of metastasis. Control mice had widespread metastasis in lymph nodes, diaphragm, pelvis, perisplenic, and perihepatic areas, whereas the incidence of metastasis was significantly lower in treated mice (Fig. 3e). Importantly, treatment with miR-409-3p mimic led to significant reduction of FABP4 protein (Fig. 3f, g) and RNA levels $(p<0.05$; Supplementary Fig. 4 e). There was no deleterious effect of the treatment on the mice weight (Supplementary Fig. $4 \mathrm{f}$ ).

Tumor microenvironmental factors in the regulation of miR-409-3p and FABP4. To identify the effects of the tumor microenvironment on FABP4 expression, we next examined various factors, including co-cultures with fibroblasts, macrophages, mesothelial cells and hypoxia treatment (Supplementary
Fig. 5a). Of the factors examined, hypoxia $\left(1 \% \mathrm{O}_{2}\right)$ consistently led to a significant increase in FABP4 expression in cancer cells at 24 and 48 h (Fig. 4a). We also examined the expression of miR409-3p after hypoxia treatment and observed low expression in hypoxic compared with normoxic cells at 24 and $48 \mathrm{~h}$ (Fig. 4b). We observed the same effect in an additional cell line (Ovca 432; Supplementary Fig. $5 b$ and c); upon exposure to hypoxia, the expression of miR-409-3p was significantly reduced, whereas FABP4 expression was significantly increased.

We then assessed the effect of hypoxia on miR409-3p expression in tumors treated with bevacizumab. This therapy, which targets vascular endothelial growth factor, has been shown to lead to hypoxia in tumors ${ }^{19}$. Hence, we compared the miR409-3p expression between bevacizumab-treated and control tumors. Expression of miR-409-3p was lower in tumors from mice treated with bevacizumab compared to control tumors (Fig. 4c). We then stained the tumor sections taken from these mice with CA9 (a hypoxia marker) and FABP4; we observed colocalization of FABP4 with CA9 (Fig. 4d). We further analyzed the TCGA and Tothill ovarian cancer datasets and observed a significant positive correlation between FABP4 and PLAU (a gene from hypoxia metagene signature) across all platforms (Fig. $4 \mathrm{e})^{20}$. The correlations of FABP4 with other genes from the metagene signature are presented in Supplementary Table 2.

Effect of FABP4 expression on protein expressions in cancer cells. To identify signaling pathways downstream of FABP4, we conducted reverse phase protein arrays (RPPA) after silencing FABP4 in HeyA8 MDR cells. The heat map of the RPPA data (Fig. 5a) showed that several proteins (Supplementary Fig. 6a and Supplementary Data 1) were downregulated after FABP4 knockdown. We further used Netwalker software to analyze the protein data, and the results showed sub pathways pertaining to metastasis being significantly downregulated in the FABP4 siRNA group (Fig. 5b).

Effect of FABP4 expression on ovarian cancer tissue metabolites. To explore the effects of FABP4 on metabolic changes in human ovarian cancer tissues, we first analyzed previously published gene expression and metabolomics data of high grade serous ovarian carcinoma tissues ${ }^{21-23}$. From those results, several metabolites were specifically expressed in either low or high FABP4 expressing patient tumor samples (Supplementary Table 3). Next, we evaluated metabolic and lipid trends related to FABP 4 gene expression by desorption electrospray ionization mass spectrometry imaging (DESI-MSI). In this study, a total of 31 high-grade ovarian cancer tissue samples were analyzed by DESI-MSI in both negative and positive ion modes to investigate a broad range of lipid and metabolites related to FABP4 expression. Using this approach, only the data specific to the tumor region were selected and evaluated, thus eliminating the inclusion of stromal lipids and metabolites.

SAM analysis (Significance Analysis of Microarrays) was conducted to identify statistically significant $\mathrm{m} / z$ values (mass to charge ratios) detected by DESI-MSI characteristic of low- or high-FABP4 expression. Positive SAM scores represent higher relative abundance in samples with low-FABP4-expression, and negative SAM scores represent higher relative abundance in samples with high-FABP4-expression. Negative ion mode data revealed 361 and 289 monoisotopic $m / z$ values associated with low or high FABP4 expression, respectively, with false discovery rate (FDR) of $<3.8 \%$. From these, $238 \mathrm{~m} / z$ values were tentatively identified by high mass accuracy measurements and tandem MS analysis (Supplementary Table 4) as small metabolites, fatty acids (FA), ceramides (Cer), glycerolipids (GL), 
a

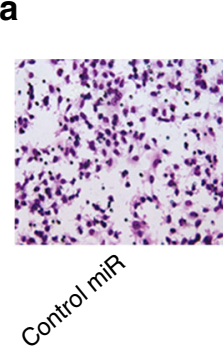

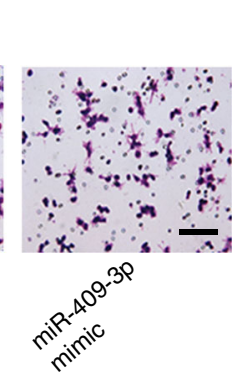
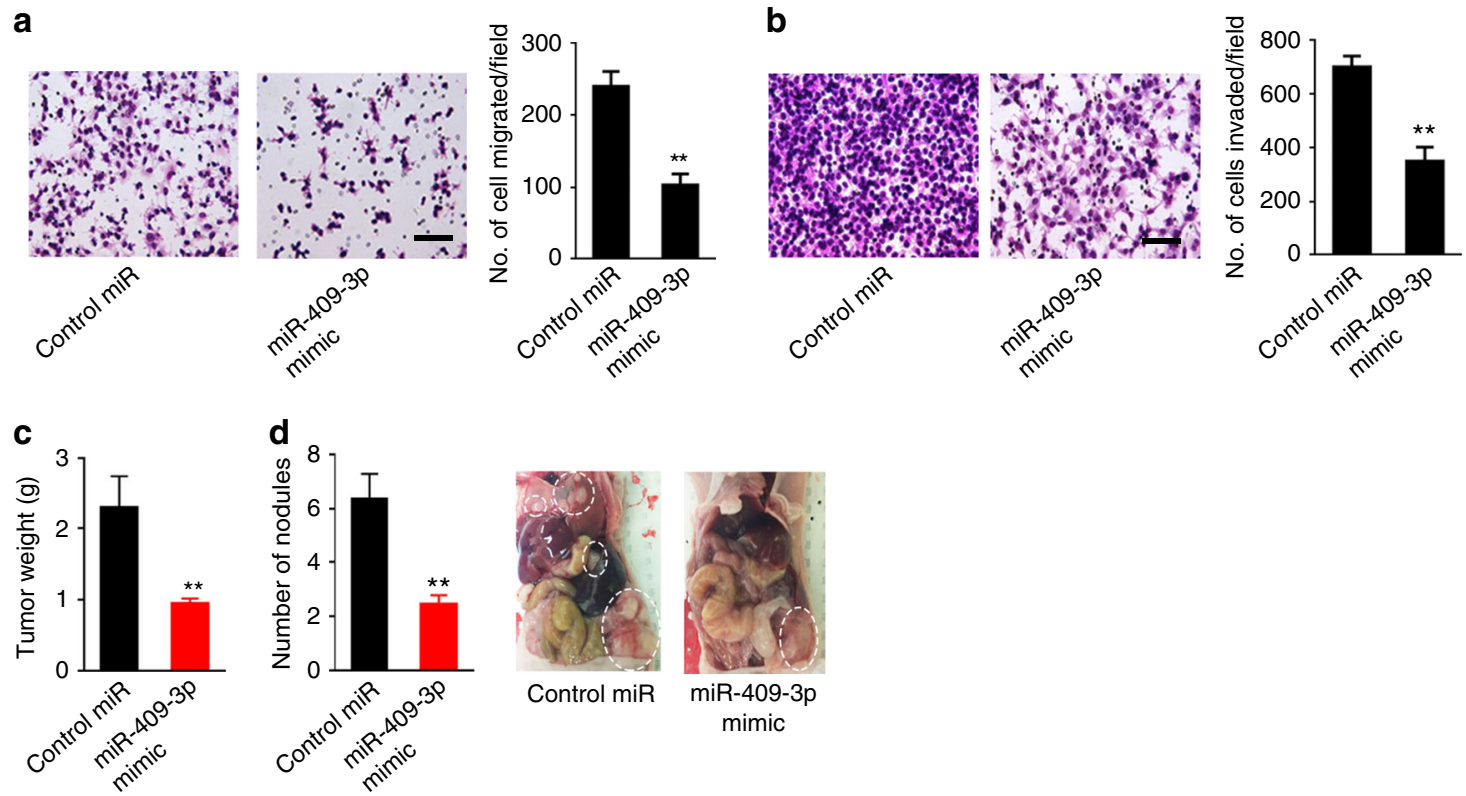

\section{e}
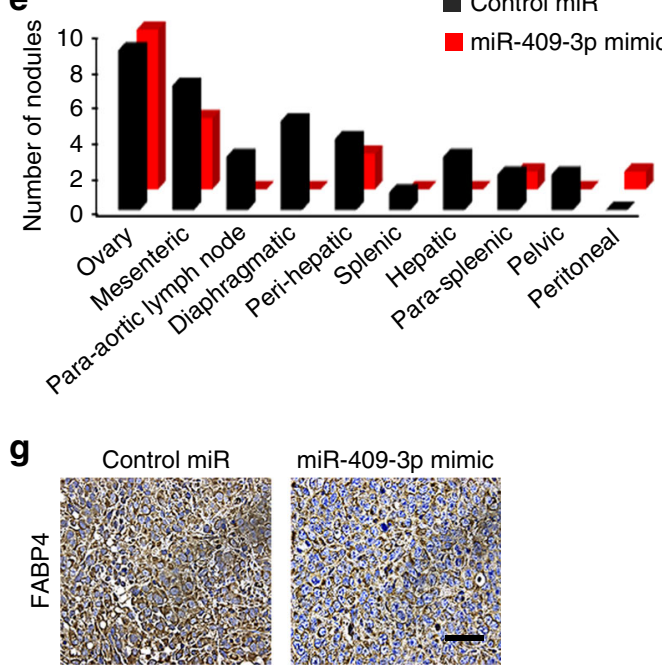

Fig. 3 Effect of miR-409-3p on tumor progression and FABP4. $\mathbf{a}$, $\mathbf{b}$ Effect of miR-409-3p mimic transfection on the $\mathbf{a}$ invasiveness and $\mathbf{b}$ migration of HeyA8 MDR cells. ${ }^{\star \star} p<0.01 . n=3$. c Aggregate tumor mass in orthotopic mouse models of ovarian cancer (HeyA8 MDR cell line) treated with control miRNA or miR-409-3p mimic incorporated in DOPC liposomes. ${ }^{\star \star} p<0.01 . n=3$. d Effect of miR-409-3p mimic on the number of nodules. Representative images are shown on the right. ${ }^{\star \star} p<0.01 . n=3$. e Distribution of tumor nodules in individual mice after treatment with control miRNA or miR-409-3p mimic. $\mathbf{f}$ Effect of treatment with miR-409-3p mimic on the expression of FABP4 (protein) in tumor samples $(n=3)$ Optical density (OD) is presented. ${ }^{\star *} p<0.01$. $\mathbf{g}$ Representative images of immunohistochemical analysis of FABP4 expression in mice treated with control miRNA or miR-409-3p mimic. Bars and errors bars represent the means and the corresponding SEMs for $n \geq 3$

monoacylglycerophosphates (PA), glycerophosphoethanolamines (PE), glycerophosphoglycerols (PG), glycerophosphoinositols (PI), glycerophosphoserines (PS), and cardiolipins (CL). Molecular formulas, detected $\mathrm{m} / z$ and mass errors in ppm are provided in Supplementary Table 5 for the species identified. Fatty acid and metabolite composition was observed to significantly differ between the two expression groups. For example, higher unsaturation and oxidation of fatty acid species was selected as characteristic of high FABP4 expression. A significant number of $\mathrm{m} / \mathrm{z}$ values related to high FABP4 expression corresponded to GL, PE, PG, and lysophospholipid species, such as LysoPE, LysoPG, and LysoPI. On the other hand, CL species were almost exclusively selected with the low-FABP4-expression type. These variations in lipid composition suggest alterations in metabolism and biosynthesis due to the changes in the expression of the FABP4 gene.

We then applied the Lasso (Least absolute shrinkage and selector operator $)^{24}$ method to evaluate if predictive statistical models could be built to classify samples as having low- or highFABP4 expression based on metabolite composition. The samples were randomly divided into a training and validation set of samples (50:50). Results based on negative ion mode data yielded an overall agreement of $81.6 \%$ and 0.79 area under the curve (AUC) value for the training set performance. The remaining independent set of samples were classified with an overall agreement of $56.1 \%$ and AUC value of 0.60 (Table 1). In positive ion mode data, an overall agreement of $74.2 \%$ for CV (AUC $=$ $0.73)$ and $61.7 \%$ agreement for the validation set $(\mathrm{AUC}=0.58)$ 

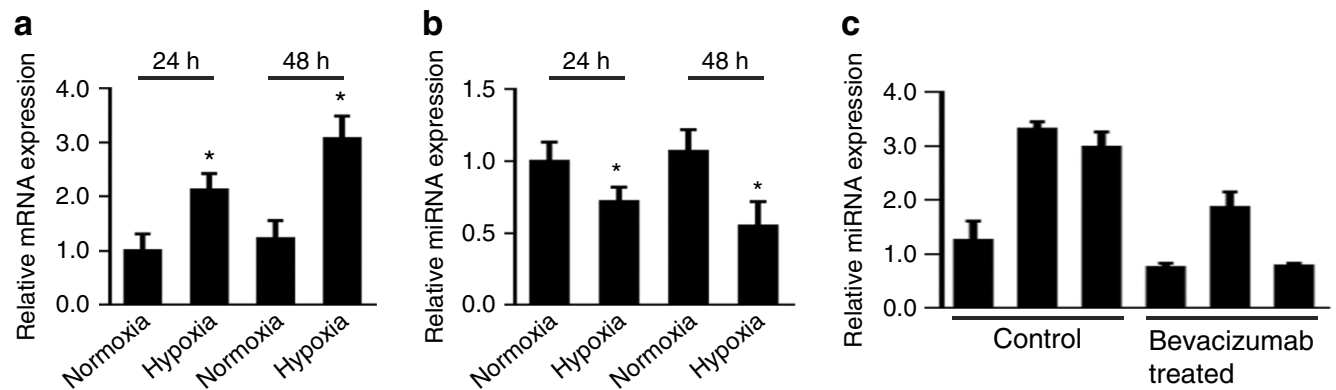
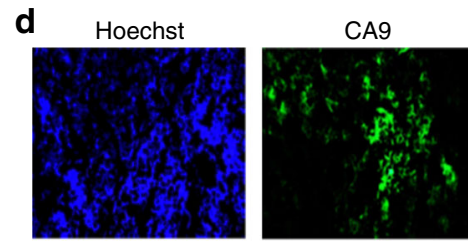

FABP4

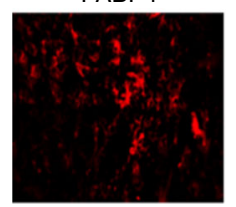

Co-localization

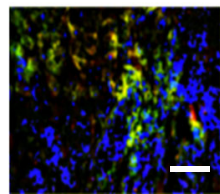

$\mathbf{e}$

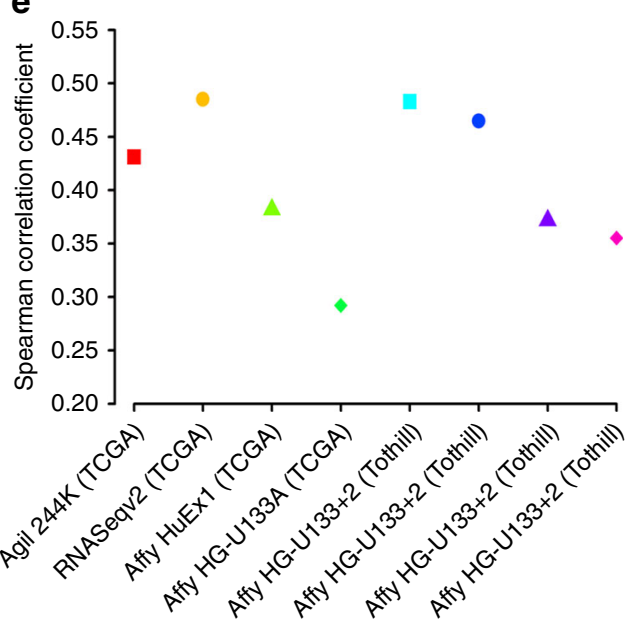

Fig. 4 Tumor microenvironmental factors in the regulation of miR-409-3p and FABP4. $\mathbf{a}$, $\mathbf{b}$ Effect of hypoxia on $\mathbf{a}$ FABP4 expression and $\mathbf{b}$ expression of miR-409-3p in HeyA8 MDR cells at $24 \mathrm{~h}$ and $48 \mathrm{~h}$. c miR-409-3p expression levels in tumor tissues from mice treated with control vehicle or bevacizumab, which is known to induce hypoxia ( $n=3$ mice per group). d Co-localization of CA9 (a hypoxia marker) and FABP4 in tumor tissues taken from mice treated with bevacizumab, which is known to induce hypoxia. Representative image is shown. e Direct correlation of FABP4 with PLAU (a hypoxia signature gene) in patient samples across different platforms. Bars and error bars represent the means and the corresponding SEMs for $n \geq 3$

(Table 1) were achieved. To improve prediction of FABP4 expression, positive and negative ion mode data were combined to build a comprehensive model. Using this approach, $66.7 \%$ of high-FABP4 patients and $100 \%$ of low-FABP4 patients were successfully classified in patient-by-patient analysis (Fig. $5 \mathrm{c}$ and Table 1).

To further investigate direct changes in lipid and metabolite levels due to the changes in FABP4 expression, we analyzed in vivo tissues from high-grade ovarian cancer mouse models presented in Fig. 1i-l. Using DESI-MSI, we compared tumor tissues of FABP4 siRNA group (low FABP4 expression, $n=3$ ) to control siRNA samples (high FABP4 expression, $n=3$ ). SAM analysis revealed 627 monoisotopic $\mathrm{m} / z$ values characteristic of FABP4 silencing, with FDR $<5.5 \%$. From the selected $m / z$ values, a total of 184 species (59 increased in low FABP4, 125 increased in high FABP4) were identified as metabolites, FA and complex lipids, with attributed SAM scores associated to FABP4 expression (Supplementary Table 6). Overall, the majority of the lipid classes received negative SAM scores, thus being associated to high FABP4 expression. For example, FA and monoacylglycerophosphates (PA) were selected exclusively for characterization of the control siRNA samples. On the other hand, Cer species were associated with low expression of the FABP4 gene. These trends were also reflected by the relative abundances observed in the ion images as shown in Fig. 5d, where all the lipid species shown except for the Cer d42:1 displayed higher relative abundances within the high FABP4 samples. Remarkably, comparison between the metabolic species selected by SAM analysis for both human and mouse samples resulted in a total of 76 common $\mathrm{m} / \mathrm{z}$ values related to high or low FABP4 expression (Table 2,
Supplementary Table 7). Among these, various FA species were associated with high expression of the FABP4 gene in both the mice and human samples. Interestingly, common GL and PI species for both animal samples were specific to the high FABP4 class, while Cer species were characteristic of tissues with low FABP4 expression. Differences in FA chain length and saturation level were also observed within the same GP class. For example, longer FA chain in PE and lysoPE species were associated with high FABP4 expression, while longer FA chain in PS lipids were characteristic to tissue samples with lower FABP4 expression. These similar metabolic trends observed in both human and mice samples strongly suggest and corroborate the effects of the FABP4 gene in metabolism and biosynthesis.

Effect of FABP4 on patient survival. High FABP4 expression was significantly correlated with poor overall (Fig. 6a) and progression-free (Fig. 6b) survival $(p<0.01)$ in the Tothill dataset $^{25}$ for both FABP4 probe sets. The multivariate analysis for overall (Supplementary Fig 6b) and progression-free (Supplementary Fig 6c) survival as well as the univariate analysis for overall (Supplementary Fig 6d) and progression-free (Supplementary Fig 6e) survival are presented.

Effect of tamoxifen on FABP4 expression and function. Having established the importance of FABP4 as a candidate target for ovarian cancer treatment, we next wished to identify clinically approved drugs that could be used to inhibit FABP4. Tamoxifen, a selective estrogen receptor modulator, has been shown to inhibit FABP4 in macrophages ${ }^{26}$. We checked whether it can be used to 
a

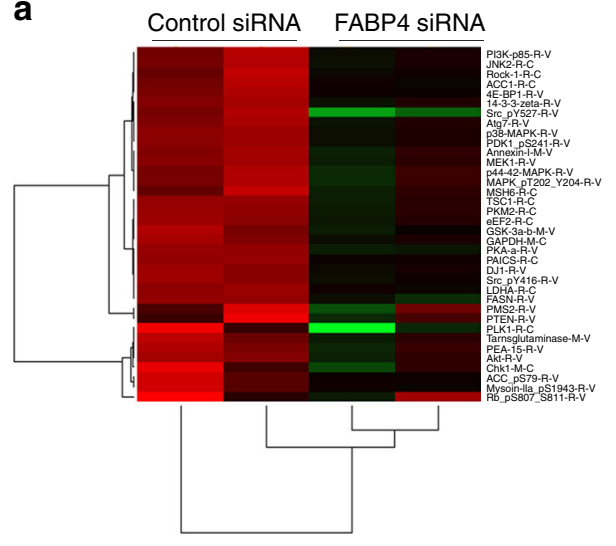

b

\begin{tabular}{|c|c|}
\hline Pathway & $p$-value \\
\hline $\begin{array}{c}\text { Microtubule bundle } \\
\text { formation }\end{array}$ & $1.96 \mathrm{E}-04$ \\
\hline $\begin{array}{c}\text { Regulation of } \\
\text { actin cytoskeleton }\end{array}$ & $3.76 \mathrm{E}-06$ \\
\hline Focal adhesion & $3.57 \mathrm{E}-09$ \\
\hline Tight junction & $1.19 \mathrm{E}-04$ \\
\hline Adherens junction & $2.04 \mathrm{E}-05$ \\
\hline
\end{tabular}

C

\begin{tabular}{|c|c|c|c|c|c|}
\cline { 3 - 6 } \multicolumn{2}{c|}{} & \multicolumn{4}{c|}{ Lasso prediction } \\
\cline { 2 - 7 } \multicolumn{1}{c|}{} & qRT-PCR & High FABP4 & Low FABP4 & Agreement (\%) & Overall agreement (\%) \\
\hline \multirow{2}{*}{ Cross-validation } & High FABP4 & 4 & 2 & 66.7 & \multirow{2}{*}{85.7} \\
& Low FABP4 & 0 & 8 & 100.0 & \\
\hline
\end{tabular}

d

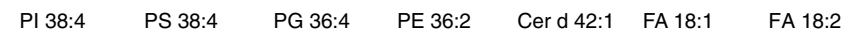

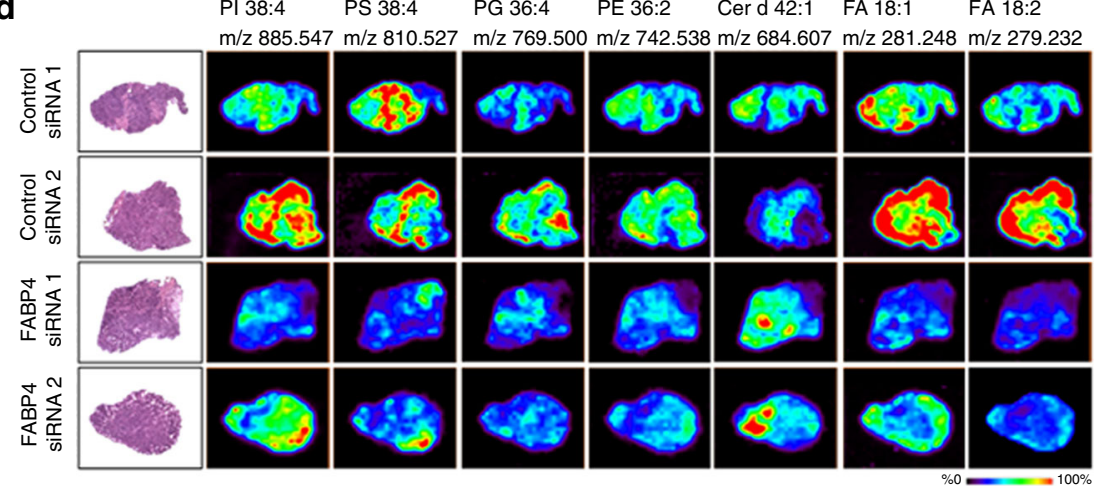

Fig. 5 Downstream effect of FABP4. a RPPA data for downregulated proteins in the FABP4 siRNA and control siRNA groups. b Netwalker analysis of RPPA data. c Model correlating molecular profiles and FABP4 expression based on the combined (negative and positive) ion mode data. $\mathbf{d}$ DESI-MS images of in vivo tumor samples from control siRNA and FABP4 siRNA treatment groups

target FABP4 in ovarian cancer cells. We used physiologically relevant concentrations of tamoxifen to determine its effect on FABP4 expression and function in ovarian cancer cells. At a concentration of $3.5 \mu \mathrm{M}$, tamoxifen decreased the expression of FABP4 by approximately $50 \%(p<0.05$; Supplementary Fig. $7 \mathrm{a})$. As a result of this downregulation, the ability of cancer cells to take up free fatty acids was also impaired. The cells treated with tamoxifen exhibited lower uptake compared to untreated control cells $(p<0.01$; Supplementary Fig. 7b). The treatment also affected the migratory potential of cancer cells and resulted in a significant decrease in the number of migrated cells (Supplementary Fig. 7c). The effect of tamoxifen on FABP4 was also observed in A2780 cells where tamoxifen treatment significantly downregulated FABP4 expression (Supplementary Fig 7d) as well as the ability of cells to migrate (Supplementary Fig $7 \mathrm{e})\left({ }^{* *} p<\right.$ $0.01)$.

\section{Discussion}

Systematically coupling bioinformatics, experimental models and clinical data, we elucidated aspects of the underlying biology of ovarian cancer that could lead to aggressive metastasis of ovarian cancer. High expression of FABP4 is a known predictor of residual disease ${ }^{4}$. Our preclinical studies show that ectopic expression of FABP4 increases tumor progression while in vivo experiments with DOPC nanoliposomes show that silencing FABP4 reduces the extent of metastasis. Our findings thus provide a promising therapeutic strategy that can potentially be useful for patients at high risk of being left with residual disease at initial surgery. Previous studies have shown that FABP4 present in the stromal cells can lead to cancer progression by providing a source of energy to cancer cells or increasing angiogenesis ${ }^{12,14}$. However, our study presents cancer cell specific FABP4 as a crucial player in promoting invasive and infiltrative metastasis in ovarian cancer. Metabolic changes in ovarian cancer have been studied before ${ }^{27,28}$. However, without spatial information, it is difficult to determine whether the metabolic alterations are occurring in the tumor or stromal compartment. To focus on the tumor compartment, we employed the DESI-MS imaging technique which allowed us to identify the chemical species present in the tissue as well as their spatial distribution. Analysis of the data, specific to tumor compartment of patient samples revealed that a unique metabolic profile is associated with the expression of FABP4. Higher unsaturation and oxidation of fatty acids and lysophospholipids were observed in higher relative abundance in samples with higher FABP4 expression. Several studies have suggested that unsaturated fatty acids play a key role in pathways leading to tumor progression by activating the beta catenin pathway, downregulating PTEN or increasing cancer cell adhesion ${ }^{29-31}$. Fatty acid oxidation has also been shown to increase metastasis in breast and ovarian cancer models ${ }^{14,32}$. Lysophospholipids have also been known to stimulate cancer cell migration and they are also considered as potential biomarkers for ovarian cancer ${ }^{33,34}$. High expression of FABP 4 can thus regulate various metabolites and protein pathways that can lead to aggressive metastasis of 
Table 1 Lasso classification of high grade serous cancer samples into high and low FABP4 expression based on DESI-MS lipid and metabolite data in negative, positive and combined polarities

\begin{tabular}{|c|c|c|c|c|c|c|c|c|}
\hline & & & \multirow[b]{2}{*}{ qRT-PCR } & \multicolumn{5}{|c|}{ Lasso prediction } \\
\hline & & & & $\begin{array}{l}\text { High } \\
\text { FABP4 }\end{array}$ & $\begin{array}{l}\text { Low } \\
\text { FABP4 }\end{array}$ & $\begin{array}{l}\text { Agreement } \\
(\%)\end{array}$ & $\begin{array}{l}\text { Overall } \\
\text { agreement (\%) }\end{array}$ & AUC \\
\hline \multirow{8}{*}{$\begin{array}{l}\text { Negative ion } \\
\text { mode }\end{array}$} & \multirow[t]{4}{*}{ For pixels } & \multirow[t]{2}{*}{ Cross-validation } & High FABP4 & 1925 & 671 & 74.2 & \multirow[t]{2}{*}{81.6} & \multirow[t]{2}{*}{0.79} \\
\hline & & & Low FABP4 & 1218 & 6450 & 84.1 & & \\
\hline & & \multirow[t]{2}{*}{ Validation set } & High FABP4 & 1003 & 1189 & 45.8 & \multirow[t]{2}{*}{56.1} & \multirow[t]{2}{*}{0.6} \\
\hline & & & Low FABP4 & 1084 & 1906 & 63.8 & & \\
\hline & \multirow[t]{4}{*}{ For patients } & \multirow[t]{2}{*}{ Cross-validation } & High FABP4 & 5 & 1 & 83.3 & \multirow[t]{2}{*}{80} & \multirow[t]{2}{*}{0.79} \\
\hline & & & Low FABP4 & 3 & 11 & 78.6 & & \\
\hline & & \multirow[t]{2}{*}{ Validation set } & High FABP4 & 4 & 2 & 66.7 & \multirow[t]{2}{*}{63.6} & \multirow[t]{2}{*}{0.6} \\
\hline & & & Low FABP4 & 2 & 3 & 60 & & \\
\hline \multirow{8}{*}{$\begin{array}{l}\text { Positive ion } \\
\text { mode }\end{array}$} & \multirow[t]{4}{*}{ For pixels } & \multirow[t]{2}{*}{ Cross-validation } & High FABP4 & 1248 & 531 & 70.2 & \multirow[t]{2}{*}{74.2} & \multirow[t]{2}{*}{0.73} \\
\hline & & & Low FABP4 & 2271 & 6823 & 75 & & \\
\hline & & \multirow[t]{2}{*}{ Validation set } & High FABP4 & 661 & 705 & 48.4 & \multirow[t]{2}{*}{61.7} & \multirow[t]{2}{*}{0.58} \\
\hline & & & Low FABP4 & 1049 & 2169 & 67.4 & & \\
\hline & \multirow[t]{4}{*}{ For patients } & \multirow[t]{2}{*}{ Cross-validation } & High FABP4 & 5 & 1 & 83.3 & \multirow[t]{2}{*}{73.7} & \multirow[t]{2}{*}{0.73} \\
\hline & & & Low FABP4 & 4 & 9 & 69.2 & & \\
\hline & & \multirow[t]{2}{*}{ Validation set } & High FABP4 & 2 & 2 & 50 & \multirow[t]{2}{*}{50} & \multirow[t]{2}{*}{0.58} \\
\hline & & & Low FABP4 & 3 & 3 & 50 & & \\
\hline \multirow{2}{*}{$\begin{array}{l}\text { Combined } \\
\text { polarities }\end{array}$} & \multirow[t]{2}{*}{ For patients } & \multirow[t]{2}{*}{ Cross-validation } & High FABP4 & 4 & 2 & 66.7 & 85.7 & - \\
\hline & & & Low FABP4 & 0 & 8 & 100 & & \\
\hline
\end{tabular}

ovarian cancer. This data elucidating the key metabolic determinants of ovarian cancer metastasis thus lay a strong foundation for future studies which can focus on the development of therapeutic strategies targeting these species.

Previous studies have identified PPAR $\gamma$ and endothelial DLL4$\mathrm{NOTCH}$ as regulatory factors for FABP $4^{11,35,36}$. Our findings provide a new understanding of FABP4 regulation by miR-4093p. MiR-409-3p has been known to target several genes such as MGMT, HMGN5 (glioblastoma) ${ }^{37,38}$, beclin, NLK and GAB1 (Colon cancer) ${ }^{39-41}$, AKT, ZEB1 (Breast cancer) ${ }^{42,43}$, c-Met (Lung cancer) $^{44}$, radixin, PHF10 (gastric cancer) ${ }^{45,46}$. In these cancer types, miR-409-3p has been known to act as a tumor suppressor whereas in prostate cancer miR-409-3p has been shown to increase EMT and stemness markers in cancer cells ${ }^{47}$. The role of miR-409-3p in ovarian cancer, however, was not known. Here, we demonstrate that miR-409-3p acts as an effective tumor suppressor in ovarian cancer through its effects on FABP4. The antitumor properties of miR-409-3p stem from its inhibitory effects on FABP4, as shown by the fact that restoration of FABP4 rescued the tumor suppressive effects of miR-409-3p on cancer cells. Importantly, we show that the DOPC nanoliposomes containing a miR-409-3p mimic effectively reduce tumor burden and metastasis in vivo. Hypoxia has been linked with ovarian cancer progression ${ }^{48,49}$. However, the exact role of hypoxia in tumor metastatic patterns was not well understood. We found that hypoxia can downregulate miR-409-3p, thus potentially removing its inhibitory effect on FABP4, which subsequently results in increased FABP4 levels. Recent findings have also suggested that HIF-1a can directly bind to the promoter region of FABP $4{ }^{50}$. The cross-talk between these regulators of FABP4 and their specific place and importance in the FABP4 pathway presents an interesting avenue for future studies.

Previous studies have shown that increased fatty acid uptake by the cells lead to increased motility and EMT ${ }^{51,52}$. Since FABP4 is a key molecule involved in free fatty acid uptake, inhibiting its ability to uptake fatty acid could be a potential therapeutic avenue to inhibit cancer metastasis. We thus identified tamoxifen as a potential drug of interest that could inhibit FABP4 and affect migration of ovarian cancer cells. Although, additional research is warranted, our data suggest tamoxifen as a promising drug for high grade serous ovarian cancer treatment, possibly in a maintenance setting.

In summary, our study provides a new understanding of a previously unrecognized mechanism (Fig. 7) by which FABP4 leads to altered tumor metastasis patterns and a higher extent of disease in ovarian cancer. Collectively, our data offer candidate targets for therapeutic interventions for ovarian cancer patients.

\section{Methods}

Cell line maintenance and siRNA and miRNA transfection. All cancer cell lines were maintained at $37^{\circ} \mathrm{C}$ and $5 \% \mathrm{CO}_{2}$ in culture with RPMI-1640 or Dulbecco's modified Eagle medium supplemented with $15 \%$ fetal bovine serum and $0.1 \%$ gentamicin sulfate (Gemini Bio-Products, Calabasas, CA, USA). HeyA8MDR, A2780, SKOV3ip1, Ovcar 5, and Ovcar 3 were received from the MDA Characterized Cell Line Core (CCLC) or directly from ATCC. Ovca 432 was received from the originator, Dr. Ronny Drapkin.

All siRNA transfections were conducted using Lipofectamine2000 as a transfecting agent. SiRNA concentration of $100 \mathrm{nM}$ was used, and the ratio of Lipofectamine (Life Technologies, Carlsbad, CA, USA) to a specific siRNA was 3:1. The cells were treated with siRNAs for $4 \mathrm{~h}$ in serum-free media before incubation in complete media for the specified time frame. For miRNA transfections (mimic and anti-miRNA), RNAiMAX (Life Technologies, Carlsbad, CA, USA was used as a transfection agent, and the ratio of RNAiMAX to a specific miRNA was 2:1. The concentration of miRNA-mimic or anti-miR inhibitor was $40 \mathrm{nM}$ concentration and the transfections were conducted in serum-free conditions. After $4 \mathrm{~h}$, the cells were incubated in complete media for the specified time frame. The sequences for siRNAs and miRNAs are listed in Supplementary Table 8.

In vivo models. Female athymic nude mice were purchased from Taconic Farms (Hudson, NY) and housed in pathogen-free conditions. The mice were cared for according to the guidelines of the American Association for Accreditation for Laboratory Animal Care International and the US Public Health Service Policy on Humane Care and Use of Laboratory Animals. All in vivo experiments and protocols were approved by MD Anderson's Institutional Animal Care and Use Committee.

To establish the tumors, $1 \times 10^{6}$ cells/mouse A2780 cells, $8 \times 10^{5}$ cells/mouse HeyA8 MDR-Luc cells, or $1 \times 10^{6}$ cells/mouse Ovcar 5 -Luc cells were injected into the ovary. No therapeutic intervention was conducted in the experiment in which FABP4-overexpressing cells were injected. For all therapeutic experiments, a siRNA or miRNA dose of $200 \mu \mathrm{g} \mathrm{kg}^{-1}$ was used, and the treatments were started 1 week after cell injections. The mice were divided into two groups: control and treatment, 10 mice/group. Mice in the control group received control siRNA or control miRNA incorporated into neutral DOPC liposomes. Mice in the treatment group 
Table 2 Lipid and metabolite species related to low and high expression of the FABP4 gene in both human and mice samples based on SAM analysis from DESI-MSI data

Metabolic species selected by SAM in both human and mice samples

siFABP4-low FABP4 expression

Control-high FABP4 expression

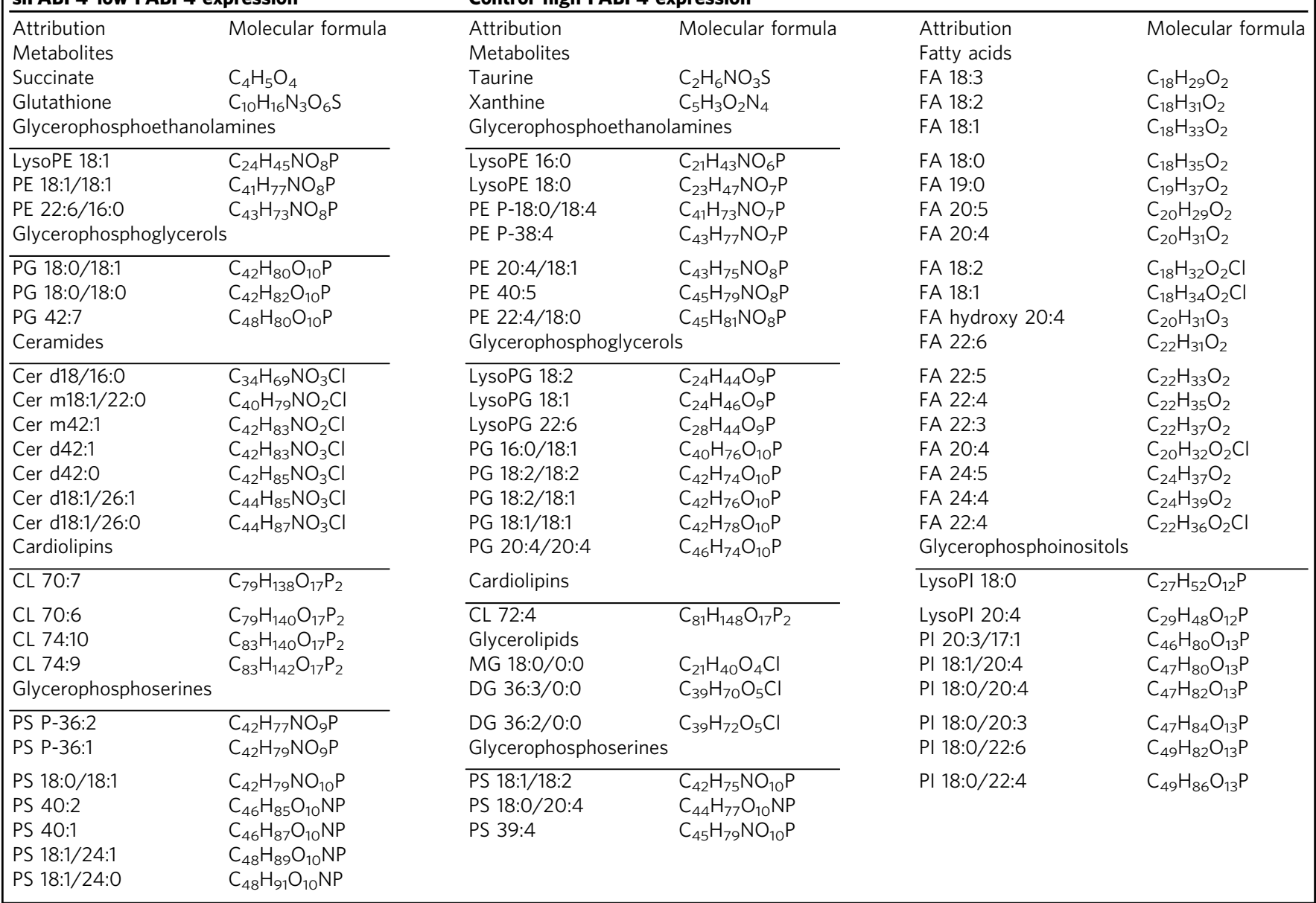

received FABP4 siRNA or a miR-409-3p mimic incorporated into DOPC liposomes. The doses were given twice weekly intraperitoneally. The mice were monitored daily for any toxic effects. Luciferase imaging was conducted to observe the effect of treatments on the metastasis as described previously ${ }^{53}$. Briefly, imaging and data acquisition were performed using the IVIS Spectrum in vivo imaging system coupled to Living Image Software (Xenogen). The mice were first anesthetized in an acrylic chamber with a mixture of $1 \%$ isoflurane. They were then injected intraperitoneally with luciferin potassium salt $\left(15 \mathrm{mg} \mathrm{ml}^{-1}\right)$ in PBS at a dose of $150 \mathrm{mg} \mathrm{kg}^{-1}$ body weight. A digital grayscale image was initially acquired, which was then overlaid with a pseudocolor image representing the spatial distribution of detected photons emerging from active luciferase present within the animal. Signal intensity was expressed as a sum of all photons detected per second. Once a mouse in any group became moribund, all mice were euthanized. Mouse weight, tumor weight, number of nodules, and locations of metastasis were recorded. Tumor tissues were then frozen in optimal cutting temperature media, fixed in formalin for paraffin embedding, or snap-frozen.

Liposomal nanoparticle preparation. Incorporation of siRNA or miRNA into DOPC liposomes was achieved as previously described ${ }^{54}$. Briefly, DOPC and siRNA or miRNA were mixed at a ratio of $1: 10(\mathrm{w} / \mathrm{w})$ in the presence of tertiary butanol. Tween 20 was added to the mixture at a ratio of 1:19. The mixture was vortexed and frozen in an acetone/dry ice bath and lyophilized. The lyophilized preparations were hydrated with $\mathrm{PBS}$ at room temperature to a concentration of $200 \mu \mathrm{g}$ of siRNA or miRNA $\mathrm{kg}^{-1}$ per injection per mouse.

MiRNA-mRNA expression association in TCGA OV samples. TCGA cases were included if the patients had high-grade disease and no history of pre-treatment. TCGA gene expression quantification were produced using justRMA applied to the CEL files from TCGA (Affymetrix HT HG-U133A arrays, $n=598$ ) $^{4}$. TCGA miRNA microarray level 3 data (Agilent $8 \times 15 \mathrm{~K}$ Human miRNA-specific microarray) was obtained from the Data Access Matrix. Data was available for 799
miRNAs. There were 541 samples meeting the inclusion criteria which had both gene expression and miRNA data available. The association between each probe for the gene of interest and each available miRNA was assessed using the Maximal Information Coefficient (MIC) computed using the publicly available MINE software of Reshef et $\mathrm{al}^{17}$.

MiRNA-mRNA Interactions. We retrieved miRNA-target interaction information from miRWalk2.0 (http://www.umm.uni-heidelberg.de/apps/zmf/mirwalk/) that hosts miRNA-target predictions from twelve programs. We selected the cases predicted by at least seven algorithms (half of the total number of programs checked +1 ). The interaction between miR-409-3p and FABP4 (NM_001442) (3'UTR) was predicted by nine programs (miRWalk, Microt4, miRanda, miRMap, miRNAMap, PITA, RNA22, RNAhybrid, Targetscan). We used Perl to sort the information available and Latex to present the sites most probable to interact.

Luciferase reporter assays, and FABP4 3'UTR site mutagenesis. Luciferase assays were conducted as described previously ${ }^{55}$. Briefly, a GoClone pLightSwitch luciferase reporter for the 3'UTR of FABP4 was purchased from Switchgear Genomics (Menlo Park, CA, USA). HeyA8 MDR cells were transfected with control miRNA or miR-409-3p mimic (100 nM; Life Technologies, Carlsbad, CA, USA) with the help of FuGENE HD transfection agent. The cells were also transfected with $3^{\prime} \mathrm{UTR}$ reporter constructs and Cypridina TK controls (pTK Cluc). After $24 \mathrm{~h}$, the LightSwitch Dual Luciferase assay kit was used and the luciferase signal was measured using a microplate luminometer, as per the manufacturer's guidelines (Biotek, Winooski, VT, USA). Luciferase activity was normalized using the Cypridina TK control, and an empty $3^{\prime} \mathrm{UTR}$ construct was used as a negative control. The ratios were then normalized to the scrambled control miRNA. Mutant FABP4 3'UTR was created for the predicted binding site mentioned in Fig. 2e using a QuickChange lightning multi-site-directed mutagenesis kit (Agilent Technologies, Santa Clara, CA, USA) using the primers mentioned in Supplementary Table 8 [AKS1] [PS2]. [SK3] The mutation was then confirmed 

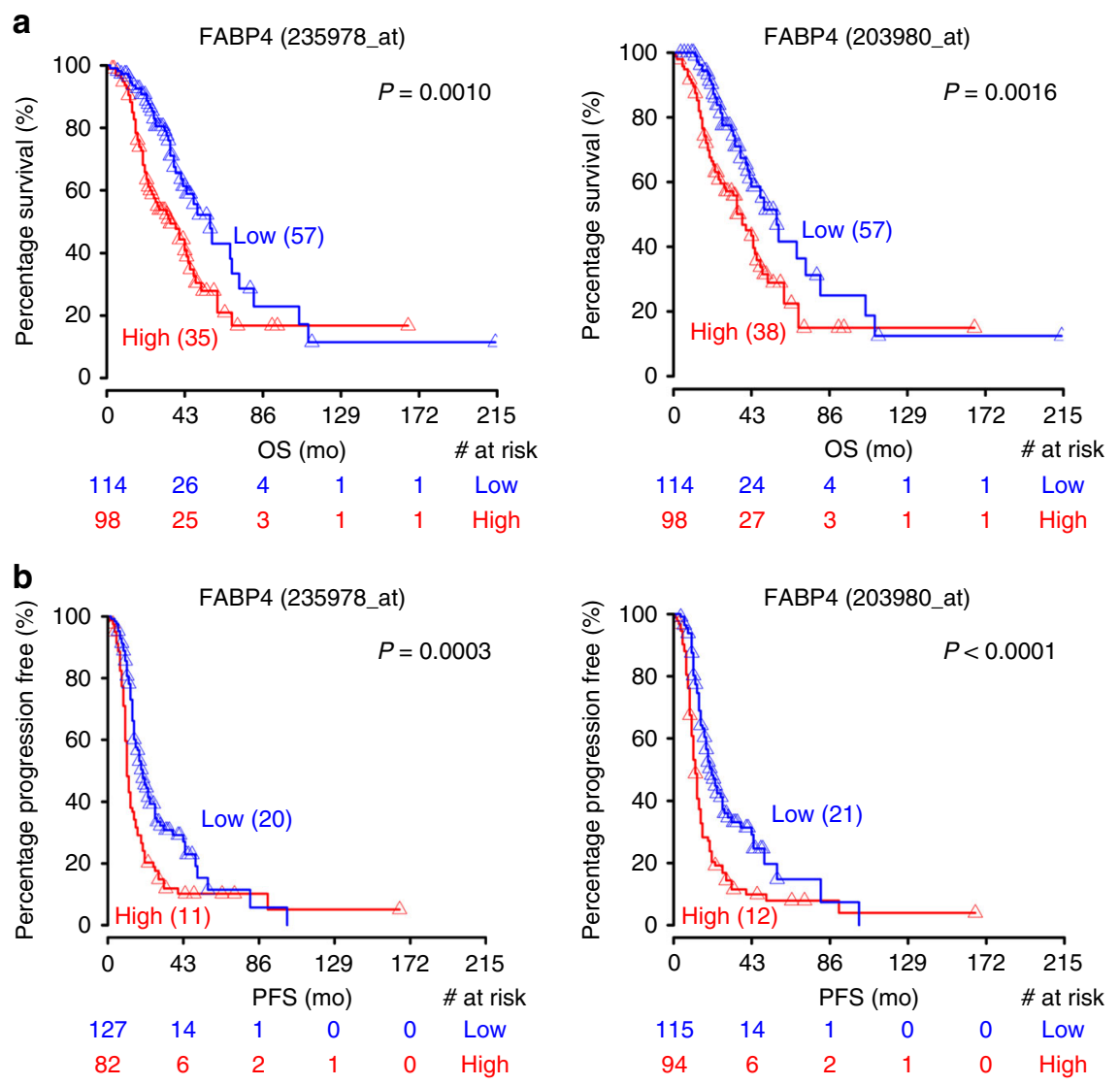

Fig. 6 Effect of FABP4 on patient survival. a, b Kaplan-Meier plots for overall (a) and progression-free (b) survival in ovarian cancer patients, based on FABP4 expression for both the probes associated with FABP4. The data were extracted from Tothill data set

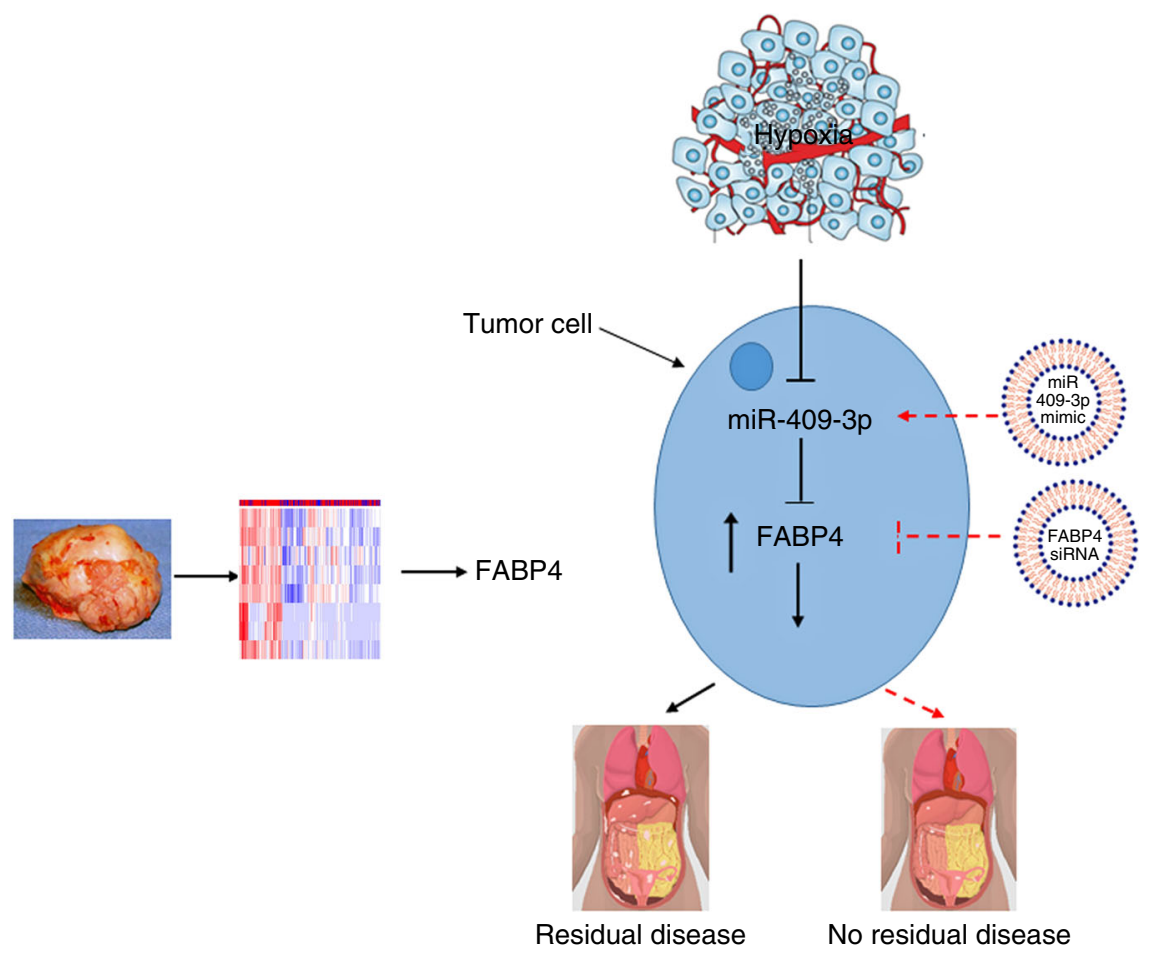

Fig. 7 Summary model. Schematic representation of regulation of FABP4, potential therapeutic strategies to target FABP4 and miR-409-3p and downstream pathological consequences of manipulating their expression 
using Sanger DNA sequencing before the mutant FABP4 3'UTR was used for the luciferase assay.

Immunoblotting. Protein lysates from tumor tissues or cultured cells were prepared using modified RIPA buffer containing proteinase and phosphatase inhibitors. Protein concentrations were determined using the BCA protein assay reagent kit (Pierce Biotechnology, Rockford, IL, USA). Lysates were loaded and separated on SDS-polyacrylamide gels. The proteins were then transferred to a nitrocellulose membrane. The membrane was blocked at room temperature for $1 \mathrm{~h}$ in $5 \%$ milk powder in Tris-buffered saline with Tween 20 (TBST) and then incubated at $4{ }^{\circ} \mathrm{C}$ overnight with primary antibodies: FABP4 (catalog number HPA002188; SigmaAldrich) and vinculin (catalog number V9131; Sigma) or beta-actin (catalog number A5316, Sigma) as loading control. After washing with TBST, the membranes were incubated with horseradish peroxidase-conjugated donkey anti-rabbit IgG (for FABP4) or sheep anti-mouse IgG (for vinculin) (catalog numbers NA934 and NA931; 1:2000; GE Healthcare) at room temperature for $2 \mathrm{~h}$. An enhanced chemiluminescence detection kit (catalog number NEL104001EA; Pierce Biotechnology) was used to visualize the horseradish peroxidase signal. Full blots are included in Supplementary Fig. 8.

Migration and invasion assay. Corning Transwell inserts (Costar, MA, USA) were coated with $0.1 \%$ gelatin (migration) or defined basement membrane matrix (invasion). Defined basement membrane matrix (for invasion) was prepared in a $10 \mathrm{ml}$ stock solution with laminin $\left(50 \mu \mathrm{g} \mathrm{ml}^{-1}\right), 1 \mathrm{ml}$ of type IV collagen $\left(50 \mu \mathrm{g} \mathrm{ml}^{-1}\right), 0.2 \mathrm{ml}$ of gelatin $\left(2 \mathrm{mg} \mathrm{ml}^{-1}\right)$, and $4 \mathrm{ml}$ and $4.8 \mathrm{ml}$ of PBS. In the upper chamber, HeyA8 MDR and Ovcar 5 cells $\left(0.7 \times 10^{5}\right)$ suspended in $200 \mu$ of serum-free media were added $48 \mathrm{~h}$ after siRNA/miRNA transfections or tamoxifen treatments. In the lower chamber, complete media for cells containing $10 \%$ fetal bovine serum $(500 \mu \mathrm{l})$ was added as a chemo-attractant. The chambers were incubated at $37^{\circ} \mathrm{C}$ in $5 \% \mathrm{CO}_{2}$ for $6 \mathrm{~h}$ (migration assay) or $24 \mathrm{~h}$ (invasion assay). After incubation, the cells in the upper chamber were removed with cotton swabs. Cells were fixed, stained, and counted using light microscopy. Cells from five random fields were counted. Experiments were done in triplicate.

Tumor samples. High-grade ovarian tumor samples were obtained from the Cooperative Human Tissue Network (CHTN) and MD Anderson Tissue Bank under an approved IRB protocol and written consent was obtained for the use of patient samples for research. The details regarding the quality control for the samples obtained from CHTN can be found at https://www.chtn.org/quality.html

miR-409-3p expression in patient tumor samples as taken from GEO dataset The detailed method can be found in Wyaman et al, $2009^{18}$. Briefly, 4 samples of HOSE (normal primary ovarian surface epithelial cells taken from postmenopausal women) and 19 serous epithelial ovarian cancer samples were analyzed. The tumor samples were high-grade and contained more than $70 \%$ malignant epithelial cellularity. The cloning frequency of a specific miR expressed as a fraction of total reads from a given sample was used to compare the relative expression of miRNAs between samples.

In situ hybridization. In situ hybridization was done as previously described ${ }^{55}$. High grade serous ovarian cancer tissues which were chemonaïve were selected. Xylene and an ethanol dilution series were used for deparaffinization and rehydration of the formalin-fixed, paraffin-embedded tissue sections. Tissue sections were digested with $15 \mu \mathrm{g} \mathrm{ml}^{-1}$ proteinase $\mathrm{K}$ for $20 \mathrm{~min}$ at room temperature and then loaded onto Ventana Discovery Ultra (Tucson, AZ) for in situ hybridization analysis. The tissue slides were then incubated with a double-digoxigenin-labeled miRCURY LNA miRNA probe (Exiqon, Woburn, MA, USA) for $2 \mathrm{~h}$ at $55^{\circ} \mathrm{C}$. Three percent $\mathrm{H}_{2} \mathrm{O}_{2}$ was used to inactivate endogenous peroxidases. After incubation with polyclonal anti-digoxigenin antibody and horseradish peroxidaseconjugated secondary antibody (Ventana), a tyramine-conjugated fluorochrome (TSA) reaction was performed for $12 \mathrm{~min}$. Sequential TSA rounds were performed for the detection of proteins using the same protocol. Slides were mounted with antifading ProLong Gold Solution (Life Technologies).

Immunostaining. Paraffin-embedded sections $(5 \mu \mathrm{m})$ of tumor tissues were cut and used for detection of FABP4. Formalin-fixed sections were deparaffinized by sequential washings with xylene, 100\% ethanol, 95\% ethanol, $80 \%$ ethanol, and PBS. After antigen retrieval, endogenous peroxidase was used to block the slides. Nonspecific binding was prevented by incubating the slides with $4 \%$ fish gelatin. This was followed by incubation with primary antibody overnight at $4{ }^{\circ} \mathrm{C}$. The next day, the slides were washed with PBS and incubated with suitable secondary antibody (goat anti-rabbit horseradish peroxidase antibody diluted in protein block solution [JB4]) for $1 \mathrm{~h}$ at room temperature. The slides were then washed with PBS followed by development with 3,3'-diaminobenzidine. The nuclei were stained with Gill's hematoxylin solution and the slides were then mounted.

For florescence staining, optimal cutting temperature-embedded frozen tissue samples were used. Nonspecific protein blocking was achieved using $4 \%$ fishgelatin solution in tris-buffered saline with tween-20. Slides were incubated with primary antibody (CA9 or FABP4) overnight at $4{ }^{\circ} \mathrm{C}$. For immunofluorescence, Alexa 594 or Alexa 499 were used for secondary antibody staining. Nuclear staining was performed using Hoechst 33342 (Molecular probes).

Quantitative real-time PCR. Total RNA was extracted from the tumor tissues using the Direct-Zol RNA extraction kit (Zymo Research, Irvine, CA, USA). RNA was then quantified using a NanoDrop spectrophotometer method and the $260 \mathrm{~nm} / 280 \mathrm{~nm}$ ratios were checked to determine quality. RNA ( $1 \mu \mathrm{g}$ per sample) was reverse-transcribed into cDNA using the Verso cDNA kit (Thermo Scientific, West Palm Beach, FL, USA) according to the manufacturer's protocol.

Quantitative real-time PCR was performed on an ABI7500 PCR system (Applied Biosystems, Warrington, UK) using $1 \mu \mathrm{l}$ of cDNA for each sample. SYBR green (Applied Biosystems) was used to detect the products, and 20 pmol of primer was used for the reaction. All reactions were carried out with $20 \mu \mathrm{l}$ of reaction mix and performed in triplicate. We used the following primers: for FABP4, 5'-TGA TGATCATGTTAGGTTTGGC-3' (forward) and 5'-TGGAAACTTGTCTCCA GTGAA-3' (reverse); for 18 S, 5'CGCCGCTAGAGGTGAAATTC3' (forward) and 5'TTGGCAAATGCTTTCGCTC3' (reverse). The following conditions were used for PCR: $50{ }^{\circ} \mathrm{C}$ for $2 \mathrm{~min}$, then $95^{\circ} \mathrm{C}$ for $15 \mathrm{~min}$, followed by 40 cycles at $95^{\circ} \mathrm{C}$ for $1 \mathrm{~min}$ each. All reactions were analyzed using the ABI7500 Applied Biosystems PCR software (v.2.0.5). The cycle threshold values of the target genes were initially normalized to the cycle threshold values of $18 \mathrm{~S}$ rRNA, and melt curves were checked to determine the specificity of the reactions.

For miRNA quantifications, Taqman miRNA assays (Life Technologies) were used and reverse-transcription real-time PCR was performed, according to the manufacturer's instructions. RNU6B was used as a housekeeping gene.

Copy number analysis. TCGA mRNA microarray (Agilent $244 \mathrm{~K}$ Custom Gene Expression G4502A-07, Affymetrix Human Genome U133A 2.0 Array, Affymetrix Human Exon 1.0 ST Array) and RNASeqv2 level 3 and clinical data were retrieved from Broad GDAC Firehose http://gdac.broadinstitute.org/. Putative copy-numbers from GISTIC were retrieved from cbio portal (http://www.cbioportal.org/). To find the relationship between FABP4 expression and copy number, we first employed a Shapiro-Wilk test and verified that the data do not follow a normal distribution. The nonparametric Kruskal-Wallis test was applied and no relationship between FABP4 and copy-number could be established. A box-andwhisker plot (Box plot represents first (lower bound) and third (upper bound) quartiles, whiskers represent 1.5 times the interquartile range) was used to visualize the data $(\log 2(\mathrm{x}))$.

Correlation of FABP4 with genes from hypoxia metagene signature. The Spearman's rank-order correlation test was applied to measure the strength of the association between FABP4 and the genes from the Winter et al. hypoxia metagene $^{20}$. We imposed a cut-off of functional relevance on the Spearman correlation coefficient in absolute value of 0.2 based on the method published previously ${ }^{56}$.

Reverse phase protein analysis. Protein lysates were isolated from HeyA8 MDR cells, $72 \mathrm{~h}$ after transfecting them with control siRNA or FABP4 siRNA. Modified RIPA buffer containing proteinase and phosphatase inhibitor was used for the lysis. Samples were then denatured by $1 \%$ SDS with $\beta$-mercaptoethanol and were analyzed by MD Anderson Core facility. The heat map was created using normalized $\log 2$ median centered values and the data was normalized using the average of the control values. The heat map was generated using http://bioinformatics. mdanderson.org/testchm/.

Correlation of FABP4 expression with metabolites present in patient tumor samples. The metabolomics data and the gene expression data (normalized log values) were taken from Zand et al., 2016 $21-23$. The gene expression was analyzed using qRT-PCR and the metabolites data was analyzed using ultrahigh performance liquid chromatography/tandem mass spectrometry (UHLC/MS/MS2) in those studies. This information was obtained from database. High grade serous ovarian cancer tumor samples $(n=61)$ were analyzed. The samples were divided into high or low FABP4 groups based on the median expression of FABP4 in all samples. Similarly, a median value for each metabolite was calculated for high or low FABP4 samples. The comparison is presented as antilog values.

DESI-MS imaging. DESI-MS imaging was conducted as described previously ${ }^{57}$. A 2D Omni Spray (Prosolia Inc., Indianapolis, IN) coupled to an LTQ-Orbitrap Elite mass spectrometer (Thermo Scientific, San Jose, CA) was used for tissue imaging. Recently, DESI-MSI has been used to characterize the metabolic profiles of ovarian high-grade serous cancer, borderline serous tumors and normal tissues, and allowed identification of potential predictive markers of ovarian cancer aggressiveness ${ }^{58}$. DESI-MS imaging was performed in the negative and positive ion mode from $m / z$ 100-1500, using a hybrid LTQ-Orbitrap mass spectrometer which allows for tandem MS experiments, high mass accuracy ( $<5 \mathrm{ppm}$ mass error), and high mass resolution $(240,000$ resolving power) measurements. The spatial resolution of the imaging experiments was $200 \mu \mathrm{m}$. Spatially accurate ion images were assembled using BioMap and MSiReader software. The histologically compatible solvent 
system dimethylformamide:acetonitrile (DMF:ACN) 1:1 ( $\mathrm{v} / \mathrm{v}$ ) was used for negative ion mode analysis, at a flow rate of $1.2 \mu \mathrm{m} \mathrm{min}^{-1}$. For positive ion mode analysis, pure ACN was used, at a flow rate of $3 \mu \mathrm{min}^{-1}$. The $\mathrm{N}_{2}$ pressure was set to 185 psi. For ion identification, high mass resolution/accuracy measurements using the same tissue sections analyzed were conducted. Tandem MS analyses were performed using both the Orbitrap and the linear ion trap for mass analysis.

Histopathology and light microscopy. The same tissue sections analyzed by DESIMS imaging were subjected afterwards to standard H\&E staining protocol. Light microscopy images of the H\&E stained slides were taken using the EVOS FL Auto Cell Imaging System (Invitrogen, Thermo Fisher Scientific, Waltham, MA, USA).

Survival analysis. Survival analysis was performed in R (version 3.2.5) using Tothill data set. In order to visualize survival (overall or progression free) differ ences between groups of patients based on FABP4 levels, we used the log-rank test to find the cut-off with the most significant (lowest $p$-value) split into high and low mRNA level groups. Kaplan-Meier plots were generated based on these cutoffs. The median survival time of each group is presented in brackets. The numbers of patients at risk in low and high mRNA groups at different time points are presented at the bottom of the graph. For overall survival, the cut-off to optimally separate the patients in the high versus low (min $p$-value) group was 0.54 for both probes associated to FABP4. For progression free survival, the cut-off to optimally separate the patients in the high versus low (min $p$-value) group was 0.55 for 203980_at and 0.61 for 235978_at.

The relationship between overall survival, respectively progression free survival and covariates (mRNA expression levels and clinical parameters (age and stage)) was examined using a Cox proportional hazard model. A multivariate Cox proportional hazard model was fitted, including the clinical parameters and mRNA expression significant in the univariate analysis.

Effect of tamoxifen on expression of FABP4. The drug was dissolved in DMSO and physiologically achievable concentrations were used for the experiments. HeyA8 MDR cells were plated in 24 well plates and serum starved before tamoxifen treatment. The cells were collected, and RNA was extracted for qRT-PCR.

Free fatty acid uptake assay. The experiment was conducted as per the manufacturer's instructions (Free Fatty Acid Uptake Assay Kit (Fluorometric, ab176768) Cambridge, MA, USA). The cells were serum starved and treated with tamoxifen at a desired concentration. Then the fatty acid dye loading solution was added $(100 \mu \mathrm{l}$ per well of a 96 well plate). The florescence signal was measured at excitation per emission 485 per $515 \mathrm{~nm}$ using a bottom read mode after $30 \mathrm{~min}$ incubation at room temperature.

Statistical analysis. MS data corresponding to the areas of interest were extracted using MSiReader software. The $m$ per $z$ range was discretized by performing hierarchical clustering and cutting the resulting dendrogram at distance 0.05 . Peaks appearing in more than $10 \%$ of the pixels were kept for analysis. Logistic regression was performed with Lasso regularization using the "glmnet" package (26) in the $\mathrm{R}$ language. Regularization parameters were determined by 3 -fold cross-validation analysis. The data were randomly equally divided into training and validation sets of samples, 50-50 per patient basis. The training set was used to build a model by cross-validation (CV) based on assigned statistical weights to specific lipid and metabolites important for classification of samples as having either high- or lowFABP4 expression. For other assays, Student's $t$-test was performed to examine the difference between the control and treatment groups. A $P$ value less than 0.05 was deemed statistically significant. All the statistical tests were two-sided. Error bars represent standard error from triplicates.

Data availability. RPPA data is provided in supplementary data. All other remaining data are available within the article and supplementary files.

Received: 13 February 2017 Accepted: 6 June 2018

Published online: 26 July 2018

\section{References}

1. du Bois, A. et al. Role of surgical outcome as prognostic factor in advanced epithelial ovarian cancer: a combined exploratory analysis of 3 prospectively randomized phase 3 multicenter trials: by the Arbeitsgemeinschaft Gynaekologische Onkologie Studiengruppe Ovarialkarzinom (AGO-OVAR) and the Groupe d'Investigateurs Nationaux Pour les Etudes des Cancers de l'Ovaire (GINECO). Cancer 115, 1234-1244 (2009).

2. Hoskins, W. J., Bundy, B. N., Thigpen, J. T. \& Omura, G. A. The influence of cytoreductive surgery on recurrence-free interval and survival in small-volume stage III epithelial ovarian cancer: a Gynecologic Oncology Group study. Gynecol. Oncol. 47, 159-166 (1992).

3. Nick, A. M., Coleman, R. L., Ramirez, P. T. \& Sood, A. K. A framework for a personalized surgical approach to ovarian cancer. Nat. Rev. Clin. Oncol. 12, 239-245 (2015).

4. Tucker, S. L. et al. Molecular biomarkers of residual disease after surgical debulking of high-grade serous ovarian cancer. Clin. Cancer Res. 20, 3280-3288 (2014)

5. Hamilton, C. A. et al. The impact of disease distribution on survival in patients with stage III epithelial ovarian cancer cytoreduced to microscopic residual: a Gynecologic Oncology Group study. Gynecol. Oncol. 122, 521-526 (2011).

6. Aragones, G. et al. Fatty acid-binding protein 4 impairs the insulin-dependent nitric oxide pathway in vascular endothelial cells. Cardiovasc. Diabetol. 11, 72 (2012).

7. Djousse, L. \& Gaziano, J. M. Plasma levels of FABP4, but not FABP3, are associated with increased risk of diabetes. Lipids 47, 757-762 (2012).

8. Holm, S. et al. Fatty acid binding protein 4 is associated with carotid atherosclerosis and outcome in patients with acute ischemic stroke. PLoS ONE 6, e28785 (2011).

9. Makowski, L., Brittingham, K. C., Reynolds, J. M., Suttles, J. \& Hotamisligil, G. S. The fatty acid-binding protein, aP2, coordinates macrophage cholesterol trafficking and inflammatory activity. Macrophage expression of aP2 impacts peroxisome proliferator-activated receptor gamma and IkappaB kinase activities. J. Biol. Chem. 280, 12888-12895 (2005).

10. Elmasri, H. et al. Endothelial cell-fatty acid binding protein 4 promotes angiogenesis: role of stem cell factor/c-kit pathway. Angiogenesis 15, 457-468 (2012).

11. Harjes, U., Bridges, E., McIntyre, A., Fielding, B. A. \& Harris, A. L. Fatty acidbinding protein 4 , a point of convergence for angiogenic and metabolic signaling pathways in endothelial cells. J. Biol. Chem. 289, 23168-23176 (2014).

12. Harjes, U. et al. Antiangiogenic and tumour inhibitory effects of downregulating tumour endothelial FABP4. Oncogene 36, 912-921 (2017).

13. Herroon, M. K. et al. Bone marrow adipocytes promote tumor growth in bone via FABP4-dependent mechanisms. Oncotarget 4, 2108-2123 (2013).

14. Nieman, K. M. et al. Adipocytes promote ovarian cancer metastasis and provide energy for rapid tumor growth. Nat. Med. 17, 1498-1503 (2011).

15. Cordero, A. B., Kwon, Y., Hua, X. \& Godwin, A. K. In vivo imaging and therapeutic treatments in an orthotopic mouse model of ovarian cancer. J. Vis. Exp. 42, 2125 (2010).

16. Greenaway, J., Moorehead, R., Shaw, P. \& Petrik, J. Epithelial-stromal interaction increases cell proliferation, survival and tumorigenicity in a mouse model of human epithelial ovarian cancer. Gynecol. Oncol. 108, 385-394 (2008).

17. Reshef, D. N. et al. Detecting novel associations in large data sets. Science $\mathbf{3 3 4}$ 1518-1524 (2011).

18. Wyman, S. K., et al. Repertoire of microRNAs in epithelial ovarian cancer as determined by next generation sequencing of small RNA cDNA libraries. PLoS ONE 4, e5311 (2009).

19. Loges, S., Mazzone, M., Hohensinner, P. \& Carmeliet, P. Silencing or fueling metastasis with VEGF inhibitors: antiangiogenesis revisited. Cancer Cell 15, 167-170 (2009).

20. Winter, S. C. et al. Relation of a hypoxia metagene derived from head and neck cancer to prognosis of multiple cancers. Cancer Res. 67, 3441-3449 (2007).

21. Zand, B. et al. Role of increased n-acetylaspartate levels in cancer. J. Natl. Cancer Inst. 108, djv426 (2016).

22. Cole, S. W. et al. Computational identification of gene-social environment interaction at the human IL6 locus. Proc. Natl. Acad. Sci. USA 107, 5681-5686 (2010).

23. Lutgendorf, S. K. et al. Depression, social support, and beta-adrenergic transcription control in human ovarian cancer. Brain Behav. Immun. 23, 176-183 (2009).

24. Tibshirani, R. Regression shrinkage and selection via the lasso. J. R. Stat. Soc. Series B Methodol., 267-288 (1996).

25. Tothill, R. W. et al. Novel molecular subtypes of serous and endometrioid ovarian cancer linked to clinical outcome. Clin. Cancer Res. 14, 5198-5208 (2008).

26. Jiang, M. et al. Tamoxifen inhibits macrophage FABP4 expression through the combined effects of the GR and PPARgamma pathways. Biochem. J. 454, 467-477 (2013).

27. Ke, C. et al. Metabolic phenotyping for monitoring ovarian cancer patients. Sci. Rep. 6, 23334 (2016).

28. Tania, M., Khan, M. A. \& Song, Y. Association of lipid metabolism with ovarian cancer. Curr. Oncol. 17, 6-11 (2010).

29. Johanning, G. L. \& Lin, T. Y. Unsaturated fatty-acid effects on human breastcancer cell-adhesion. Nutr. Cancer 24, 57-66 (1995). 
30. Kim, H. et al. Unsaturated fatty acids stimulate tumor growth through stabilization of beta-catenin. Cell Rep. 13, 495-503 (2015).

31. Vinciguerra, M. et al. Unsaturated fatty acids promote hepatoma proliferation and progression through downregulation of the tumor suppressor PTEN. J. Hepatol. 50, 1132-1141 (2009).

32. Park, J. H. et al. Fatty acid oxidation-driven Src links mitochondrial energy reprogramming and oncogenic properties in triple-negative breast cancer. Cell Rep. 14, 2154-2165 (2016).

33. Monet, M. et al. Lysophospholipids stimulate prostate cancer cell migration via TRPV2 channel activation. Biochim. Et. Biophys. Acta 1793, 528-539 (2009).

34. Sutphen, R. et al. Lysophospholipids are potential biomarkers of ovarian cancer. Cancer Epidemiol., Biomark. Prev. Publ. Am. Assoc. Cancer Res. cosponsored Am. Soc. Prev. Oncol. 13, 1185-1191 (2004).

35. Boiteux, G. et al. A-FABP, a candidate progression marker of human transitional cell carcinoma of the bladder, is differentially regulated by PPAR in urothelial cancer cells. Int. J. Cancer 124, 1820-1828 (2009).

36. Khalil, S. et al. miRNA array screening reveals cooperative MGMT-regulation between miR-181d-5p and miR-409-3p in glioblastoma. Oncotarget 7, 28195-28206 (2016).

37. Cao, Y., Zhang, L., Wei, M., Jiang, X. \& Jia, D. MicroRNA-409-3p represses glioma cell invasion and proliferation by targeting high-mobility group nucleosome binding domain 5. Oncol. Res. 20 1097-1107 (2017).

38. Tan, S. et al. miR-409-3p sensitizes colon cancer cells to oxaliplatin by inhibiting Beclin-1-mediated autophagy. Int J. Mol. Med. 37, 1030-1038 (2016).

39. Liu, M. et al. Downregulation of microRNA-409-3p promotes aggressiveness and metastasis in colorectal cancer: an indication for personalized medicine. J. Transl. Med. 13, 015-0533 (2015).

40. Bai, R. et al. MicroRNA-409-3p suppresses colorectal cancer invasion and metastasis partly by targeting GAB1 expression. Int. J. Cancer 137, 2310-2322 (2015).

41. Zhang, G., Liu, Z., Xu, H. \& Yang, Q. miR-409-3p suppresses breast cancer cell growth and invasion by targeting Akt1. Biochem. Biophys. Res. Commun. 469, 189-195 (2016).

42. Ma, Z. et al. MicroRNA-409-3p regulates cell invasion and metastasis by targeting ZEB1 in breast cancer. IUBMB Life 68, 394-402 (2016).

43. Wan, L. et al. MicroRNA-409-3p functions as a tumor suppressor in human lung adenocarcinoma by targeting c-Met. Cell Physiol. Biochem 34, 1273-1290 (2014).

44. Zheng, B. et al. MicroRNA-409 suppresses tumour cell invasion and metastasis by directly targeting radixin in gastric cancers. Oncogene 31, 4509-4516 (2012).

45. Li, C. et al. MicroRNA-409-3p regulates cell proliferation and apoptosis by targeting PHF10 in gastric cancer. Cancer Lett. 320, 189-197 (2012).

46. Josson, S. et al. miR-409-3p/-5p promotes tumorigenesis, epithelial-tomesenchymal transition, and bone metastasis of human prostate cancer. Clin. Cancer Res. Off. J. Am. Assoc. Cancer Res. 20, 4636-4646 (2014).

47. Rupaimoole, R. et al. Hypoxia-mediated downregulation of miRNA biogenesis promotes tumour progression. Nat. Commun. 5, 5202 (2014).

48. van den Beucken, T. et al. Hypoxia promotes stem cell phenotypes and poor prognosis through epigenetic regulation of DICER. Nat. Commun. 5, 5203 (2014).

49. Hu, B. et al. Fatty acid binding protein-4 (FABP4) is a hypoxia inducible gene that sensitizes mice to liver ischemia/reperfusion injury. J. Hepatol. 63, 855-862 (2015).

50. Balaban, S. et al. Adipocyte lipolysis links obesity to breast cancer growth: adipocyte-derived fatty acids drive breast cancer cell proliferation and migration. Cancer Metab. 5, 1 (2017).

51. Nath, A., Li, I., Roberts, L. R. \& Chan, C. Elevated free fatty acid uptake via CD36 promotes epithelial-mesenchymal transition in hepatocellular carcinoma. Sci. Rep. 5, 14752 (2015).

52. Lee, J. W. et al. EphA2 immunoconjugate as molecularly targeted chemotherapy for ovarian carcinoma. J. Natl Cancer Inst. 101, 1193-1205 (2009).

53. Landen, C. N. Jr. et al. Therapeutic EphA2 gene targeting in vivo using neutral liposomal small interfering RNA delivery. Cancer Res. 65, 6910-6918 (2005).

54. Rupaimoole, R. et al. Hypoxia-upregulated microRNA-630 targets Dicer, leading to increased tumor progression. Oncogene 35, 4312-4320 (2016).
55. Cancer Genome Atlas Research, N. Integrated genomic analyses of ovarian carcinoma. Nature 474, 609-615 (2011).

56. Wiseman, J. M., Ifa, D. R., Song, Q. \& Cooks, R. G. Tissue imaging at atmospheric pressure using desorption electrospray ionization (DESI) mass spectrometry. Angew. Chem. Int. Ed. Engl. 45, 7188-7192 (2006).

57. Eberlin, L. S. et al. Molecular assessment of surgical-resection margins of gastric cancer by mass-spectrometric imaging. Proc. Natl Acad. Sci. USA 111 2436-2441 (2014).

58. Sans, M. et al. Metabolic markers and statistical prediction of serous ovarian cancer aggressiveness by ambient ionization mass spectrometry imaging. Cancer Res. 77, 2903-2913 (2017).

\section{Acknowledgements}

This work was supported, in part, by the National Institutes of Health (CA177909, CA016672, CA213759, UH3TR000943, P50 CA217685, P50 CA098258, R35 CA209904, and R00CA190783), Cancer Prevention and Research Institute of Texas (RP110595, RP120214), Ovarian Cancer Research Fund, Inc. (Program Project Development Grant), the RGK Foundation, The Judi A. Rees ovarian cancer research fund, Mr. and Mrs. Daniel P. Gordon, The Blanton-Davis Ovarian Cancer Research Program, the American Cancer Society Research Professor Award, and the Frank McGraw Memorial Chair in Cancer Research (A.K.S.). K.M.G. was supported by Altman Goldstein Discovery Fellowship. S.P. was supported by Ovarian Cancer Research Fund Alliance and Foundation for Women's Cancer. S.Y.W. was supported by Ovarian Cancer Research Fund Alliance, Foundation for Women's Cancer, Colleen Dream Foundation, and CPRIT training grants (RP101502 and RP101489). This work was also supported by P30CA016672 and used the Bioinformatics Shared Resource.

\section{Author contributions}

K.M.G. designed the study and performed experiments, analyzed data and wrote the manuscript. S.P. participated in in vivo studies and manuscript preparation. S.P., R.R., S.Y.W., A.S.N., X.Z., M.H., M.M., C.M., X.C., M.A.T., A.V., G.A.P., and R.K. participated in in vitro and in vivo studies. M.S. and L.E. helped with DESI-MS imaging analysis. C.I., S.T., and K.B. helped with statistical analysis and patient datasets. P.K. and P.R. helped with Netwalker analysis. J.L. helped with H\&E analysis. L.S.M., C.R, G.L.B. designed and prepared DOPC nanoparticles. R.N. helped with the model figure. A.K.S designed and supervised the study and participated in manuscript preparation. All authors edited and approved the final manuscript.

\section{Additional information}

Supplementary Information accompanies this paper at https://doi.org/10.1038/s41467018-04987-y.

Competing interests: The authors declare no competing interests.

Reprints and permission information is available online at http://npg.nature.com/ reprintsandpermissions/

Publisher's note: Springer Nature remains neutral with regard to jurisdictional claims in published maps and institutional affiliations.

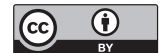

Open Access This article is licensed under a Creative Commons Attribution 4.0 International License, which permits use, sharing, adaptation, distribution and reproduction in any medium or format, as long as you give appropriate credit to the original author(s) and the source, provide a link to the Creative Commons license, and indicate if changes were made. The images or other third party material in this article are included in the article's Creative Commons license, unless indicated otherwise in a credit line to the material. If material is not included in the article's Creative Commons license and your intended use is not permitted by statutory regulation or exceeds the permitted use, you will need to obtain permission directly from the copyright holder. To view a copy of this license, visit http://creativecommons.org/ licenses/by/4.0/.

(C) The Author(s) 2018 